\title{
A Cryptochrome adopts distinct moon- and sunlight states and functions as moonlight interpreter in monthly oscillator entrainment
}

\author{
Short title: Moonlight sets a monthly clock via L-Cry
}

\begin{abstract}
Birgit Poehn ${ }^{\star 1,2}$, Shruthi Krishnan³, Martin Zurl1,2, Aida Coric ${ }^{\# 1,2}$, Dunja Rokvic ${ }^{\# 1,2}$, Enrique Arboleda ${ }^{1,2,4}$, Lukas Orel1,2, Florian Raible ${ }^{1,2}$, Eva Wolf ${ }^{@ 3}$ and Kristin Tessmar-Raible ${ }^{@ 1,2}$

${ }^{1}$ Max Perutz Labs, University of Vienna, Vienna BioCenter, Dr. Bohr-Gasse 9/4, 1030 Vienna
\end{abstract}

${ }^{2}$ Research Platform "Rhythms of Life", University of Vienna, Vienna BioCenter, Dr. Bohr-Gasse 9/4, A1030 Vienna

${ }^{3}$ Structural Chronobiology, Institute of Molecular Physiology (IMP) and Institute of Molecular Biology (IMB) Mainz, Ackermannweg 4, 55128 Mainz, Germany.

${ }^{4}$ present address: Institut de Génomique Fonctionnelle de Lyon (IGFL), École Normale Supérieure de Lyon, 32 avenue Tony Garnier, 69007 Lyon, France

*,\# equal contribution

@ correspondence:

Eva Wolf: evawolf1@uni-mainz.de

Kristin Tessmar-Raible: kristin.tessmar@mfpl.ac.at 


\section{Abstract}

Measuring time by the moon's monthly cycles is a wide-spread phenomenon and crucial for successful reproduction in countless marine organisms. In several species, such as the mass-spawning bristle worm Platynereis dumerilii, an endogenous monthly oscillator synchronizes reproduction to specific days. Classical work showed that this oscillator is set by full moon. But how do organisms recognize this specific moon phase? We uncover L-Cry's involvement: photoreduction and recovery kinetics of its co-factor FAD differ strongly when purified L-Cry is exposed to naturalistic moonlight, naturalistic sunlight, or their different successions. L-Cry's sun- versus moonlight states correlate with distinct sub-cellular localizations, indicating differential signalling. These properties enable a discrimination between sunand moonlight, as well as moonlight duration as a moon phase indicator. Consistently, l-cry mutants re-entrain their circalunar phase less well than wild-type to naturalistic moonlight. But under artificially strong nocturnal light, I-cry mutants re-entrain faster than wildtype, suggesting that L-Cry at least partly blocks "wrong" light from impacting on this oscillator. Our work provides a new level of functional understanding of moon-regulated biological processes. 


\section{Main text}

The moon fascinates biologists and non-biologists alike. An impact of lunar light on the nervous system, and ultimately on the behavior and metabolism of animals, may at first seem eccentric to modern scientists. But as a matter of fact, lunar influences on animals are especially well documented in the marine environment (1-3). Starting with the early 20th century, numerous scientific studies have shown that the reproductive behavior and sexual maturation of animals as diverse as corals, polychaetes, echinoderms, fishes or turtles are synchronized by the lunar cycle ( 1 , 3-7). A recent study suggests that the lunar cycle coordinates the behaviour of a nocturnal migratory bird (8), and recently uncovered clear correlations of human sleep and menstrual cycle properties with moon phases have re-initiated the discussion of an impact of the moon even on human biology $(9,10)$. In animals, these synchronizations are not only impressive, but typically also important to ensure successful reproduction (11). In turn, desynchronization of these reproductively critical rhythms due to anthropogenic disturbances, as documented for corals, poses a threat to species survival (11).

Despite the importance and widespread occurrence of lunar rhythms, any functional mechanistic insight is lacking. Importantly, this synchronization among conspecifics is in many cases not simply a direct reaction to a stimulus, but instead governed by endogenous monthly oscillators: circalunar clocks $(3,12-16)$. The marine bristle worm Platynereis dumerilii is well-documented to possess such a circalunar clock, which controls its reproductive timing and can be entrained by nocturnal light in the lab $(5,14,17)$. Several reports have linked the expression of cryptochromes (CRYs) with moon phase, suggesting that these genes could be involved in circalunar time-keeping (18), possibly- as proposed for corals- as lunar light receptors $(2,19,20)$. However, no functional molecular support for such an involvement exists. In order to move from expression correlation to a mechanistic understanding, we investigated the biochemical properties and functional role of the light-receptive cryptochrome L-Cry in the annelid Platynereis dumerilii (14).

\section{L-Cry responds to naturalistic moonlight by cumulative photoreduction}

While we have previously shown that Pdu-L-Cry is degraded upon light exposure in S2 cell culture (14), it has remained unclear if L-Cry has the spectral properties and sensitivity to sense moonlight and whether this would differ from sunlight sensation. To test this, we purified full-length L-Cry from insect cells (figs. S1A-C). Multiangle light scattering (SEC-MALS) analyses of purified dark-state L-Cry revealed a molecular weight of $133 \mathrm{kDa}$, consistent with the predicted molecular homodimer weight of 135 kDa (Fig. 1A). Purified L-Cry binds Flavin Adenine Dinucleotide (FAD) as its chromophore (figs. S1D,E). We then used UV/Vis absorption spectroscopy to analyze the FAD photoreaction of L-Cry. The 
bioRxiv preprint doi: https://doi.org/10.1101/2021.04.16.439809; this version posted April 18, 2021. The copyright holder for this preprint (which was not certified by peer review) is the author/funder, who has granted bioRxiv a license to display the preprint in perpetuity. It is made available under aCC-BY-NC-ND 4.0 International license.
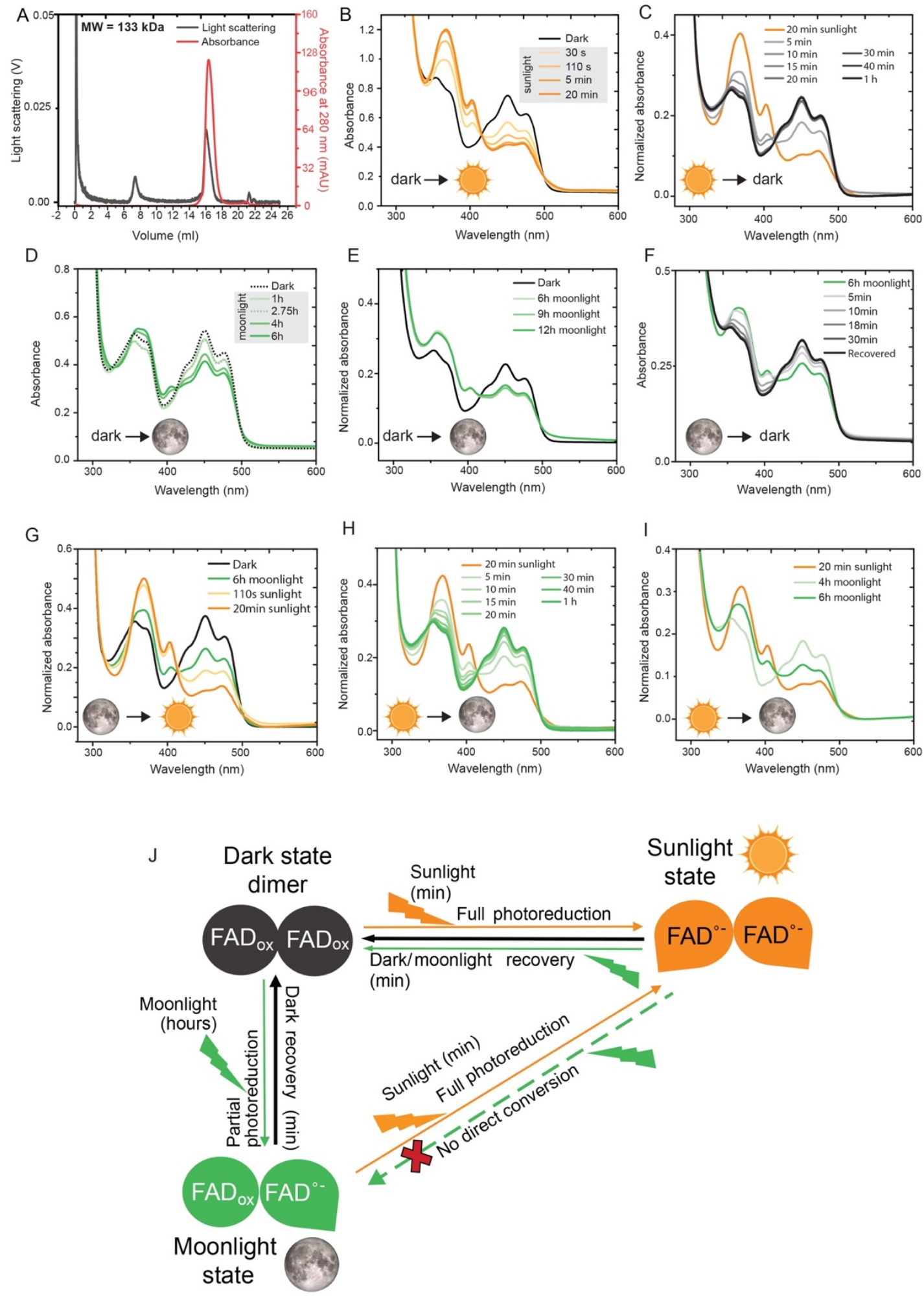

Figure 1: L-Cry is differently photoreduced by sunlight and moonlight.

(A) Multi-Angle Light Scattering (MALS) analyses of dark-state L-Cry supports L-Cry homodimer formation (theoretical MW $135 \mathrm{kDa}$ ). (B) Absorption spectrum of L-Cry in darkness (black) and after sunlight exposure (orange). Additional timepoints: S2A. (C) Dark recovery of L-Cry after $20 \mathrm{~min}$ sunlight on ice. Absorbance at $450 \mathrm{~nm}$ in S2B (D,E) Absorption spectra of L-Cry after exposure to naturalistic moonlight for different durations. (F) Full spectra of dark recovery after $6 \mathrm{~h}$ moonlight. Absorbance at 450nm: S2D. (G) Absorption spectrum of L-Cry after $6 \mathrm{~h}$ of moonlight followed by $20 \mathrm{~min}$ of sunlight. (H) 
Absorption spectrum of L-Cry after 20min sunlight followed by moonlight first results in dark-state recovery. Absorbance at 450nm: S2E. (I) Absorption spectrum of L-Cry after $20 \mathrm{~min}$ sunlight followed by $4 \mathrm{~h}$ and $6 \mathrm{~h}$ moonlight builds up the moonlight state. (J) Schematic model of Pdu L-Cry responses to sun- and moonlight. MALS data (A) and preliminary SAXS analyses (data not shown) suggest that L-Cry forms homodimers in its dark and sunlight states, also see discussion and fig. S2I.

As basic starting point to analyze its photocycle, L-Cry was photoreduced with a $445 \mathrm{~nm}$ emitting strong blue light LED (fig. S3D) for 110s (21). The light-activated spectrum showed that blue-light irradiation of $L-C r y$ leads to the complete conversion of $F A D_{\text {ox }}$ into an anionic $F A D$ radical $\left(F A D^{\circ}\right)$ with characteristic $F A D^{\circ-}$ absorption maxima at $370 \mathrm{~nm}$ and $404 \mathrm{~nm}$ and reduced absorbance at $450 \mathrm{~nm}$ (fig. S1F, blue spectrum, black arrows). In darkness, L-Cry reverted back to the dark state with time constants of $2 \min \left(18^{\circ} \mathrm{C}\right), 4 \min \left(6^{\circ} \mathrm{C}\right)$ and $4.7 \mathrm{~min}$ (ice) (figs. S1G-K ).

We then investigated the response of L-Cry to ecologically relevant light, i.e. sun- and moonlight using naturalistic sun- and moonlight devices we designed based on light measurements at the natural habitat of Platynereis dumerilii (22) (figs. S3A,C,E). Upon naturalistic sunlight illumination, $F A D$ was photoreduced to $F A D^{\circ}$, but with slower kinetics than under the blue light source, likely due to the intensity differences between the two lights (figs. S3C-E). While blue-light illumination led to a complete photoreduction within 110 s (fig. S1F), sunlight-induced photoreduction to $F A D^{\circ-}$ was completed after $20 \mathrm{~min}$ (Fig. 1B) and did not further increase upon continued illumation for up to $2 \mathrm{~h}$ (fig. S2A). Dark recovery kinetics had time constants of $3.2 \mathrm{~min}\left(18^{\circ} \mathrm{C}\right)$ and $5 \mathrm{~min}$ (ice) (Fig.1C, fig. $\mathrm{S} 2 \mathrm{~B}, \mathrm{C})$.

As the absorbance spectrum of L-Cry overlaps with that of moonlight at the Platynereis natural habitat (figs. S3A), L-Cry has the principle spectral prerequisite to sense moonlight. However, the most striking characteristic of moonlight is its very low intensity $\left(1.79 \times 10^{10}\right.$ photons $/ \mathrm{cm}^{2} / \mathrm{s}$ at $-5 \mathrm{~m}$, figs. S3A,E). To test if $P d u$-L-Cry is sensitive enough for moonlight, we illuminated purified L-Cry with our custom-built naturalistic moonlight, closely resembling full moon light intensity and spectrum at the Platynereis natural habitat (figs. S3A,C,E). Naturalistic moonlight exposure up to 2.75 hours did not markedly photoreduce FAD, notably there was no difference between 1 hours and 2.75 hours (Fig. 1D). However, further continuous naturalistic moonlight illumination of $4 \mathrm{~h}$ and longer resulted in significant changes (Fig. 1D), whereby the spectrum transitioned towards the light activated state of $F A D^{\circ-}$ (note peak changes at $404 \mathrm{~nm}$ and at $450 \mathrm{~nm}$ ). This photoreduction progressed further until 6 h naturalistic moonlight exposure (Fig. 1D,E). No additional photoreduction could be observed after 9 $\mathrm{h}$ and $12 \mathrm{~h}$ of naturalistic moonlight exposure (Fig.1E), indicating a distinct state induced by naturalistic moonlight that reaches its maximum after $\sim 6 \mathrm{hrs}$, when about half of the L-CRY molecules are photoreduced. This time of $\sim 6 \mathrm{hrs}$ is remarkably consistent with classical work showing that an additional $6 \mathrm{hr}$ of nocturnal light period are important for circalunar clock entrainment, irrespective of the preceding photoperiod (5). The dark recovery of L-Cry after $6 \mathrm{~h}$ moonlight exposure occurred with a time constant of $6.7 \mathrm{~min}$ at $18^{\circ} \mathrm{C}$ (Fig. 1F, fig. S2D). Given that both sunlight and moonlight 
cause FAD photoreduction, but with different kinetics and apparent different final $F A D^{\circ-}$ product/FAD ox adduct ratios, we wondered how purified L-Cry would react to transitions between naturalistic sun- and moonlight (i.e. during "sunrise" and "sunset").

Mimicking the sunrise scenario, L-Cry was first illuminated with naturalistic moonlight for $6 \mathrm{~h}$ followed by $20 \mathrm{~min}$ of sunlight exposure. This resulted in an immediate enrichment of the $F A D^{\circ-}$ state (Fig. 1G). Hence, naturalistic sunlight immediately photoreduces remaining oxidized flavin molecules, that are characteristic of moonlight activated $L-C R Y$, to $F A D^{\circ}$, to reach a distinct fully reduced sunlight state.

In contrast, when we next mimicked the day-night transition ("sunset") by first photoreducing with naturalistic sunlight (or strong blue light) and subsequently exposed L-Cry to moonlight, L-Cry first returned to its full dark state within about $30 \mathrm{~min}$ (naturalistic sunlight: $\tau=7 \mathrm{~min}$ (ice): Fig.1H, fig.S2E; blue light: $\tau=9 \min$ (ice): fig. S2F-H), despite the continuous naturalistic moonlight illumination. Prolonged moonlight illumination then led to the conversion of dark-state L-Cry to the "moonlight state" (Fig. 1I, fig. S2F ), i.e. fully photoreduced "sunlight- state" L-Cry first has to return into the dark state before entering the "moonlight- state" characterized by the stable presence of the partial FAD product/FAD ox adduct. In contrast to "sunlight- state" L-Cry, "moonlight-state" L-Cry does not return to the oxidized ("dark") state under naturalistic moonlight. Taken together, these results indicate the existance of kinetically and structurally distinct "sunlight" and "moonlight" states of L-Cry (Fig.1J, S2I).

\section{Naturalistic sun- and moonlight differently affect L-Cry subcellular localization}

To further study the function of L-Cry in Platynereis, we generated two I-cry mutant alleles ( $\triangle 34$ and $\triangle 11 \mathrm{bp}$ ) (Fig.2A) using TALENS (23), as well as a monoclonal antibody against Pdu-L-Cry. Using the mutant worms, we first verified the specificity of the anti-L-Cry antibody in Western blot (Fig. 2B) and immunohistochemistry (Fig. 2E-J). Furthermore, we verified that the staining of the antibody in wildtype worms (Fig. 2E-H) matches the regions where l-cry mRNA is expressed (Fig. 2D). These tests confirmed the absence of L-Cry protein in mutants and the specificity of the anti-L-Cry antibody. 
bioRxiv preprint doi: https://doi.org/10.1101/2021.04.16.439809; this version posted April 18, 2021. The copyright holder for this preprint (which was not certified by peer review) is the author/funder, who has granted bioRxiv a license to display the preprint in perpetuity. It is made available under aCC-BY-NC-ND 4.0 International license.

A

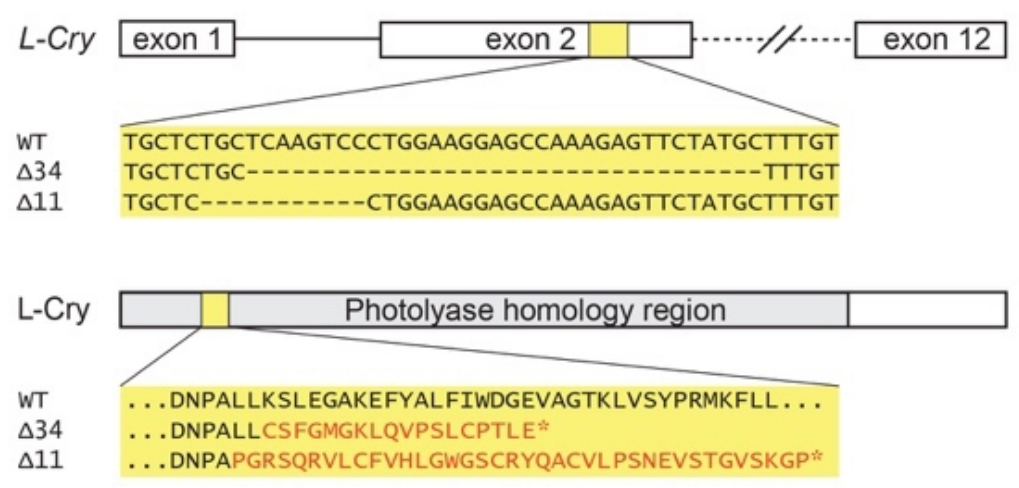

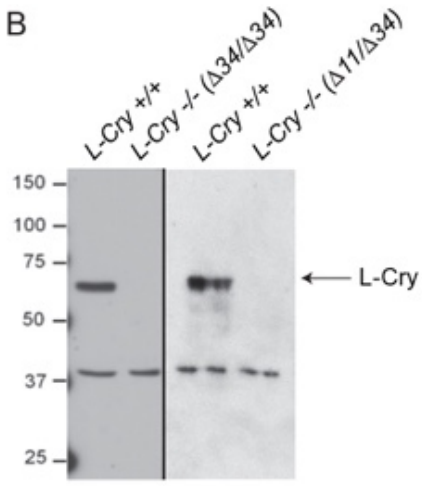

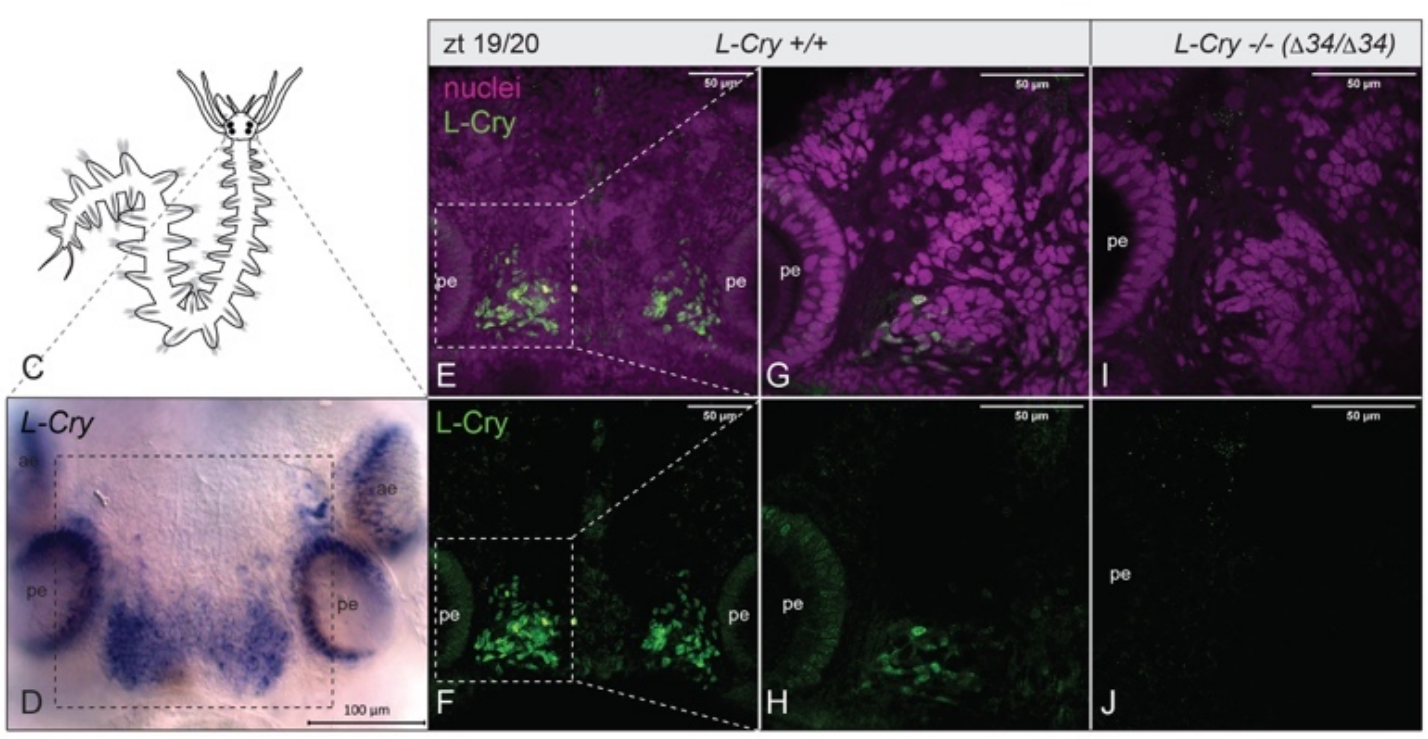

Figure 2. I-cry -/- mutants are loss-of-function alleles

(A) Scheme of the L-Cry genomic locus for wt and mutants. Both mutant alleles result in an early frameshift and premature stop codon. The $\Delta 34$ allele has an additional $9 \mathrm{bp}$ deletion in exon 3. (B) Western Blots of $P$. dumerilii heads probed with anti-L-Cry antibody. (C) scheme of $P$. dumerilii. (D) whole mount in situ hybridization against I-cry mRNA on worm head. ae, anterior eye; pe, posterior eye. (E-J) Immunohistochemistry of premature wildtype (E,F,G,H) and mutant (I,J) worm heads sampled at zt19/20 using anti-L-Cry antibody (green) and Hoechst staining (magenta), dorsal views, anterior up. E,F: z-stack images (maximal projections of 50 layers, $1.28 \mu \mathrm{m}$ each) in the area highlighted by the rectangle in (D), whereas (G-J) are single layer images of the area highlighted by the white rectangles in $(E)$ and $(F)$.

In order to further investigate the response of L-Cry to naturalistic sun- and moonlight, we conducted Western blots and immunohistochemistry at different lunar and daily timepoints (Figs. 3A-A"). L-Cry levels after 8 h of naturalistic sunlight (day before full moon = FM-1, diel time: zeitgeber time $8=z t 8$, Figs. $\left.3 A, A^{\prime}\right)$ were significantly reduced compared to $8 \mathrm{~h}$ under darkness at the same moon phase (FM1, zt 24, Figs. 3B,C), in line with (canonical) L-Cry degradation in response to naturalistic sunlight. 
bioRxiv preprint doi: https://doi.org/10.1101/2021.04.16.439809; this version posted April 18, 2021. The copyright holder for this preprint (which was not certified by peer review) is the author/funder, who has granted bioRxiv a license to display the preprint in perpetuity. It is made available under aCC-BY-NC-ND 4.0 International license.

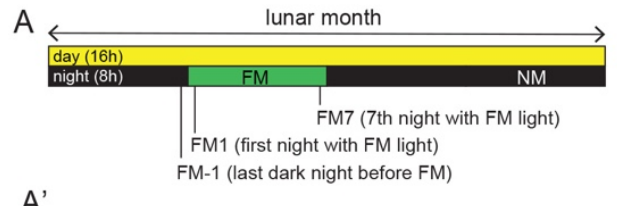

$A^{\prime}$

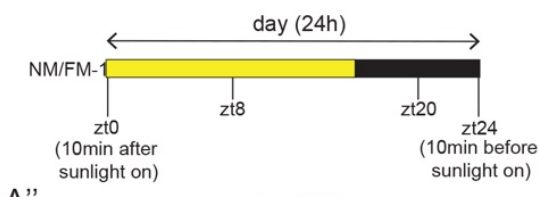

A"

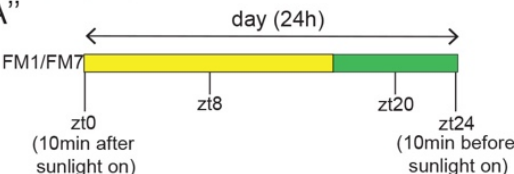

D

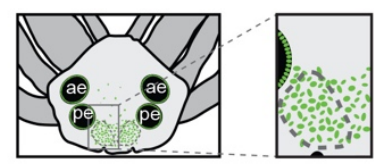

- cytoplasmic

$\leqslant$ nuclear
B

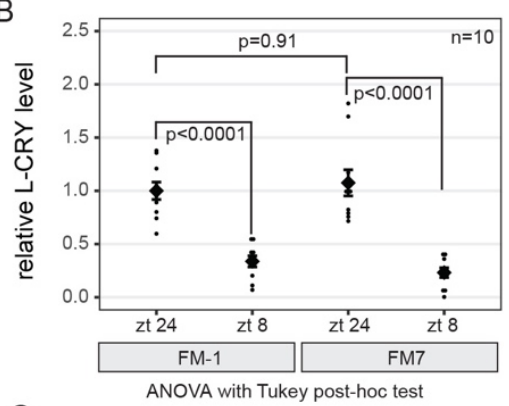

C

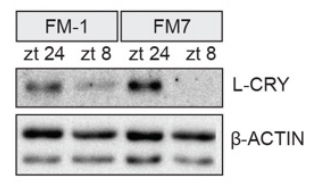

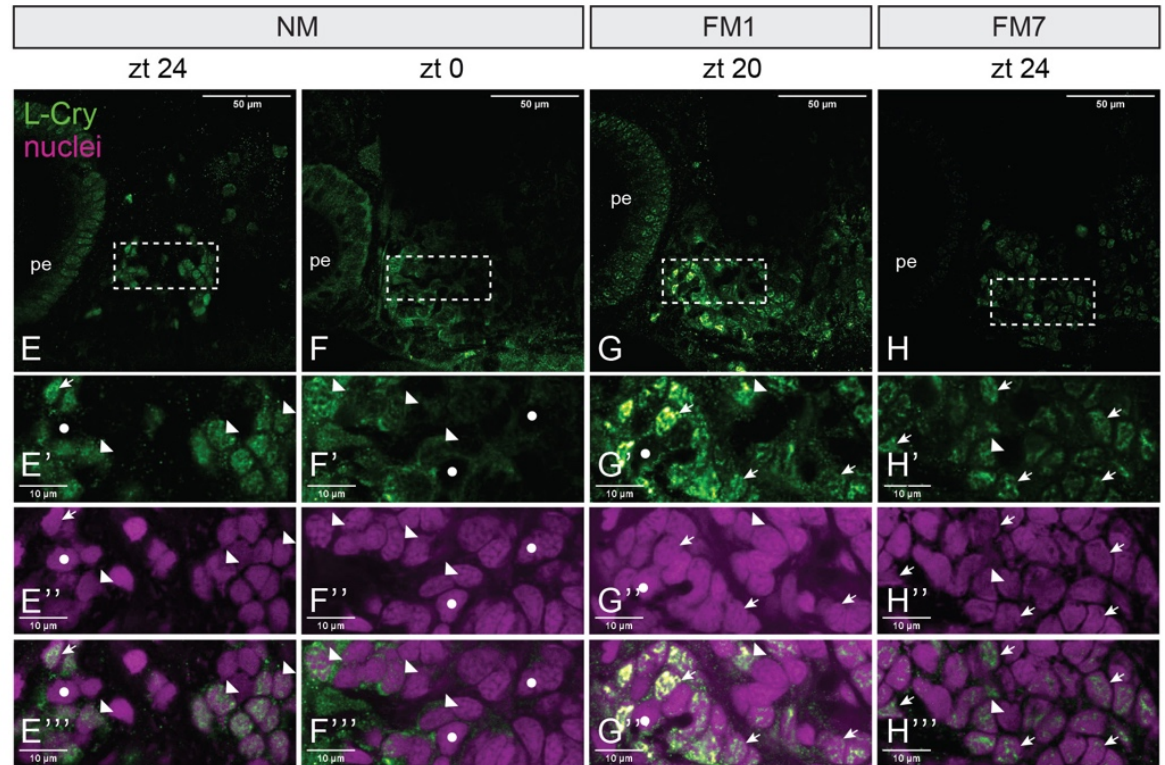

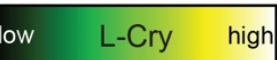

both (cytoplasmic + nuclear)

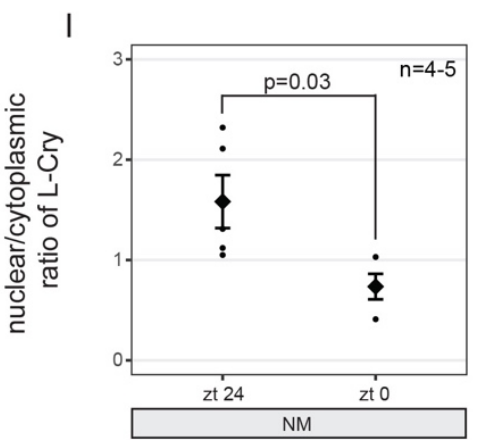

$\mathrm{J}$

Figure 3: Naturalistic moon- and sunlight impact differently on L-Cry localization and levels.

$\left(\mathbf{A}, \mathbf{A}^{\prime}, \mathbf{A}^{\prime \prime}\right)$ Scheme of sampling timepoints. $16 \mathrm{hrs}$ day and $8 \mathrm{hrs}$ night per $24 \mathrm{hrs}, 8$ nights of moonlight per month. (B) relative $\mathrm{L}$-Cry levels at indicated timepoints, as determined by Western Blot. Individual data points as well as mean \pm SEM are shown. (C) Representative Western Blot used for quantification in (B), see fig.S9 for all other. (D) P.dumerilii head scheme. Dashed ovals designate the oval-shaped posterior domains between the posterior eyes. Green dots: L-Cry+ cells. ae, anterior eye; pe, posterior eye. (E-H) Maximal projections of confocal images of worm heads stained with anti-L-Cry antibody (green) and HOECHST (magenta: nuclei). White rectangles: areas of the zoom-ins presented below. zoomed pictures: single layers $(1.28 \mu \mathrm{m})$. Arrows: nuclear L-Cry, dots: cytoplasmatic L-Cry, arrowheads: both cytoplasmic and nuclear 
nuclear/cytoplasmic ratio at indicated timepoints. Individual data points as well as mean \pm SEM are shown. $p$-values: twotailed t-test. For quantification as categorical data, see fig.S4E-G, S8.

In contrast to sunlight, exposure of L-Cry to $8 \mathrm{~h}$ of naturalistic moonlight did not cause a reduction in L-Cry levels compared to an equivalent time in darkness (FM-1, zt24 versus FM7, zt 24: Figs.3B,C). Thus, any potential moonlight signalling via L-Cry occurs via a mechanism independent of L-Cry degradation.

We next examined the spatial regulation of L-Cry in worm heads (scheme Fig. 3D) at lunar and diel timepoints (Fig. 3A-A"). At NM, zt24, L-Cry is found predominantly in both nucleus and cytoplasm of individual cells, (Fig. 3E-E'”, quantification as numerical data, i.e. nuclear/cytoplasmic ratio: Fig. 3I,J, for quantification as categorical data (24): fig.S4E-G). Already after $10 \mathrm{mins}$ of exposure to naturalistic sunlight (NM zt0, Fig. 3A, $A^{\prime}$ ), the L-Cry nuclear localization strongly diminishes, becoming increasingly only cytoplasmic (Fig. 3F-F"', numerical quantification Fig. 3I, categorical quantification fig.S4E,F, also see accompanying manuscript Zurl et al). This suggests that naturalistic sunlight causes a shift of the protein to the cytoplasm, followed by degradation (FM-1, zt8, Fig. 3B,C, also see accompanying manuscript Zurl et al).

On the first night lit by the naturalistic moonlight for circalunar entrainment, L-Cry is strongly present at zt20, a timepoint which is consistent with having sufficient time to biochemically respond to naturalistic moonlight and to reach its moonlight-state (FM1, zt20, Fig. 3A, $\left.A^{\prime \prime}, G-G^{\prime \prime \prime}\right)$, thus fulfilling a prerequisite for moonlight sensation. Upon further naturalistic moonlight exposure for seven continuous nights (FM7, zt24) L-Cry remained clearly nuclear (Fig. 3H-H'"', numerical quantification Fig. 3J, categorical quantification: fig.S4G). Thus, the sunlight and moonlight-states of L-Cry correlate with clearly distinct subcellular distribution patterns. In fact, we observed that L-Cry at FM7, zt24 is even more nuclear restricted than at zt24 under NM, both in the numerical analysis of the nuclear/cytoplasmic ratio (Fig. 3J), as well as in the blind categorical scoring (fig.S4G). This suggests that also the dark and moonlight states of L-Cry have distinct subcellular distribution patterns.

Taken together, these findings show that moonlight and sunlight impact differentially on L-Cry quantity and localization. The translocation of L-Cry from nucleus to cytoplasm in response to sunlight followed by reduced L-Cry levels suggests canonical degradation of L-cry in the cytoplasm. In contrast, moonlight does not cause L-Cry degradation, but might further shift its subcellular localization towards the nucleus.

\section{L-cry mutants entrain less well than wildtype to naturalistic moonlight} As we found that L-Cry can respond to naturalistic moonlight biochemically, is present at night when moonlight can occur and responds differently to naturalistic moon vs. sunlight, we next wondered, if the circalunar clock responds differently to naturalistic moonlight in Platynereis wt versus I-cry-/worms. We performed a circalunar re-entrainment experiment: animals initially raised and entrained 
bioRxiv preprint doi: https://doi.org/10.1101/2021.04.16.439809; this version posted April 18, 2021. The copyright holder for this preprint (which was not certified by peer review) is the author/funder, who has granted bioRxiv a license to display the preprint in perpetuity. It is made available under aCC-BY-NC-ND 4.0 International license.

This re-entraining stimulus was repeated for three consecutive months (Fig. 4A).

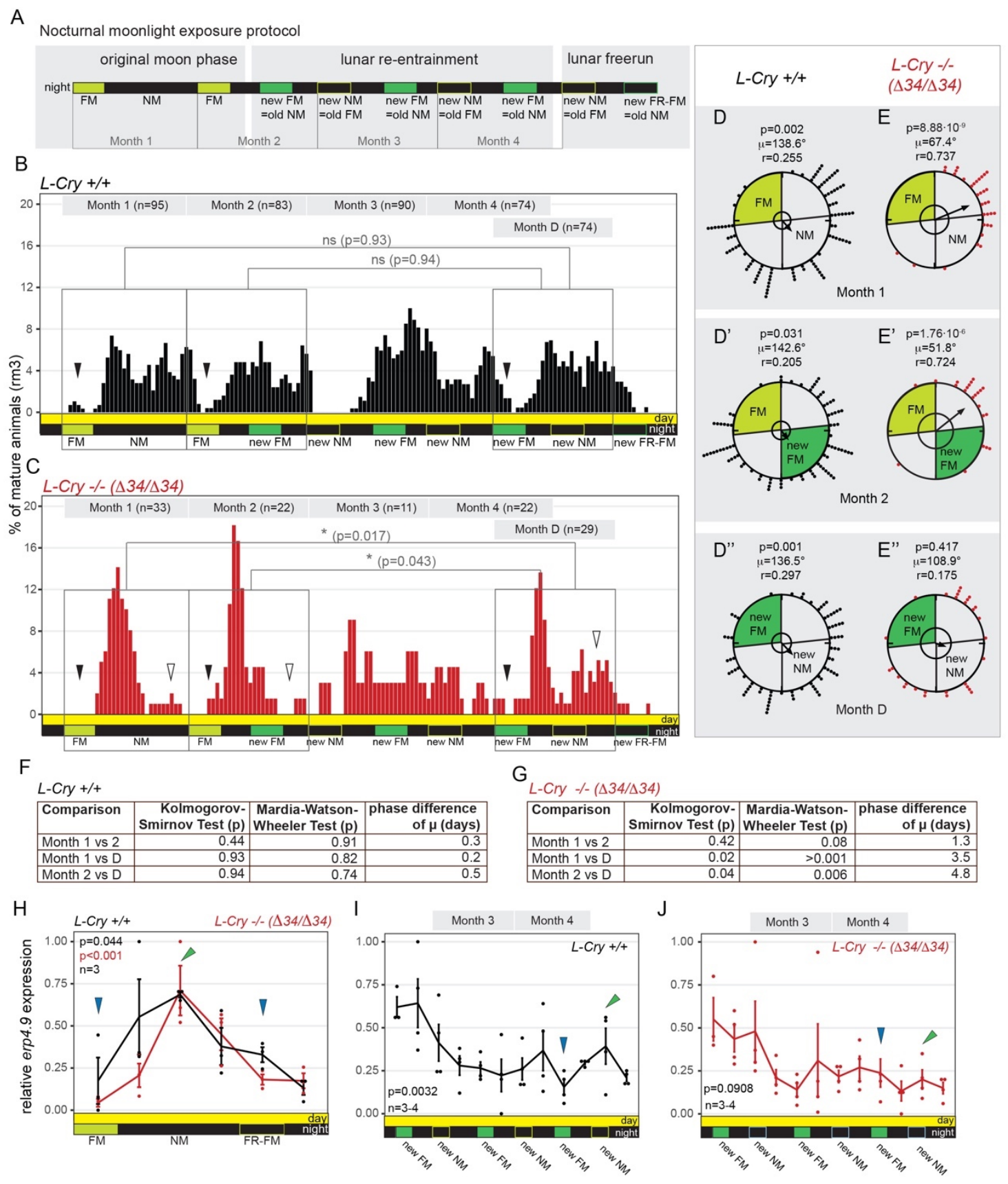

Figure 4: I-cry contributes to circalunar clock entrainment

(A) Nocturnal moonlight exposure protocol of lunar phase shift with 8 nights of naturalistic moonlight. (B,C) Number of mature animals (percent per month, rolling mean with a window of 3 days) of $I$-cry wildtype (B) and mutant (C) animals. pvalues: Kolomogorov-Smirnov tests. Black arrowheads indicate spawning-free intervals of the wildtype. White arrowheads in (C) designate the increasing second peak in the l-cry mutant after re-entrainment. (D,E) Data as in (B,C) plotted as circular data. $360^{\circ}$ correspond to 30 days of the lunar month. The arrow represents the mean vector $\mu$. $r$ (length of $\mu$ ) indicates phase coherence (measure of population synchrony). p-values are results of Rayleigh Tests: Significance indicates nonrandom distribution of data points. The inner circle represents the Rayleigh critical value $(p=0.05)$. $(F, G)$ Results of multisample statistics on spawning data shown in (A-E). (H) Circalunar regulation of erp4.9 transcript levels in wildtype and mutant animals (heads of immature animals, lab condition, 6 nights of full moon). Individual data points as well as mean \pm SEM are shown. p-values: one-way ANOVA. (I,J) Expression of the lunar regulated gene erp4.9 in premature heads of wildtype (I) and I-cry mutants (J) during the lunar phase shift experiment. p-values: one-way ANOVA. 
The resulting maturation peaks were analysed for the efficacy of this "naturalistic moonlight" to phase-shift the circalunar oscillator. In order to judge how each population responded, we compared the spawning distribution of the months before with the last month of the phase shift for each genotype.

For an explanation of the noticeable difference in spawning distribution comparing identical months between genotypes, see Fig. 5 and corresponding section below.

Under the re-entrainment regime (Fig. 4A), wildtype animals completely shifted their spawning pattern, as supported by two statistical analyses:

(1) No significant difference in Kolmogorov-Smirnov test when comparing months 1 and 2 before the shift to the newly entrained last month (month D) of the shift (Fig. 4B: grey rectangles, Fig. 4F);

(2) no significant difference in phase distribution with circular analyses of the same data when comparing the months before the shift with the last month of the shift (Fig. 4D, D' versus $D^{\prime \prime}, F$ ). Phase coherence (indicated as vector length $r$, a measure of population synchrony), was also very similar before and in the last month of the shift $r=0.255$ and $r=0.297$ ) and the resulting phase difference was 0.2 and 0.5 days, respectively (Fig. 4F).

In contrast, the spawning distribution of I-cry mutants shifted less than that of their wildtype counterparts based on the same criteria:

(1) Kolmogorov-Smirnov testing showed significant differences in spawning distribution when comparing months 1 and 2 before the shift to the last month of the shift (Fig. 4C: grey rectangles, Fig. $4 \mathrm{G)}$;

(2) a significant difference in the phase distribution in the circular analyses when comparing the months before the shift with the last month of the shift (Fig. 4E, $E^{\prime}$ versus $\left.E^{\prime \prime}, G\right)$. The population also exhibited a much lower phase coherence in the last month of the shift $(r=0.175)$ than before the shift $(r=0.737)$, and a noticeable phase difference of 3.5 and 4.8 days, respectively (Fig. $4 \mathrm{G}$ ).

Consistent with a less coherent shift of the I-cry-/- mutant population, we observed a pronounced second peak in the last month of the phase shift, likely representing a subpopulation of worms that still obeyed the old lunar phase (Fig. 4C, compare white arrowheads). A similar relative increase of worms in the second part of the lunar months was not noticeable for the wildtype population (Fig. $4 B)$.

In order to verify our phase shift results with an independent assay, we analyzed a molecular readout. We had previously identified erp4.9 as being regulated by the circalunar clock on transcript and protein level (25). qPCR analyses after long-term monthly entrainment validated erp4.9 regulation across the lunar month in both wildtype and mutant worms with identical phase and amplitude (Fig. $4 \mathrm{H}$ ), and thus suitable for phase comparisons between wildtype and mutant worms in re- 
entrainment analyses. We started the molecular analysis at the first new full moon and subsequently sampled every week at zt6. Initially, both wildtype and mutants showed high expression (as expected for the original NM phase), likely representing the situation that most worms were still entrained to the original lunar phase (Fig. $4 \mathrm{I}, \mathrm{J}$ ). The levels of erp4.9 for wildtype and mutants subsequently lowered and remained low for several weeks, possibly representing populations of worms with mixed circalunar phases (Fig. $4 \mathrm{I}, \mathrm{J})$. Importantly, during the third re-entrainment month, erp4.9 levels in wildtype animals indicated a shift of the circalunar clock, with low levels exactly matching the new FM, and peak levels matching new NM (compare blue and green arrowheads black curve Fig. $4 \mathrm{H}$ vs. I). By contrast, in the l-cry -/- population, such a profile was not detectable (compare blue and green arrowheads red curve Fig. $4 \mathrm{H}$ vs. J).

Taken together, both spawning data and erp4.9 expression dynamics suggest that I-cry mutant populations entrain less well to naturalistic moonlight than wildtype, providing evidence that L-Cry is part of the circalunar entrainment pathway.

\section{l-cry mutants show altered lunar spawning synchrony}

\section{Another phenotype that is obvious from the above spawning data is that homozygous $1-\mathrm{cry}^{-/-}$animals} grown under conventional culture light conditions (i.e. artificial sun- and moonlight fig.S3B) showed a higher population synchrony $(r)$ than wildtype or heterozygous animals, exhibiting sharper spawning peaks (Fig. 5A-C). This difference between mutants, wildtype and heterozygous animals is statistically significant (Fig. 5A-C, Kolmogorov-Smirnov-Test on overall data distribution, Fig. 5I) and also well visible in the circular analyses (Fig. 5E-G): Wildtype siblings and heterozygous worms distribute their spawning across a lunar month significantly different from random (Rayleigh test $p=1.75 \times 10^{10}$ and $\left.p=3.13 \times 10^{8}\right)$, but with rather low phase coherence, i.e. population synchrony $(r=0.295$ and $r=0.222)$, consistent with the wildtype data from the phase shift experiment (Fig. 4B,D-D"). In contrast, homozygous 1 -cry ${ }^{-/-}$mutants exhibit a high phase coherence $(r=0.614)$, consistent with the mutant data from the phase shift experiment (Fig. 4C, E-E'). The differences between mutant and wildtype/heterozygous controls are also highly significant by the Mardia-Watson-Wheeler test on the circular data (Fig. 5I). The specificity of this phenotype of higher spawning precision for mutations in the I-cry gene was confirmed by analyses on trans-heterozygous I-cry ${ }^{(\Delta 34 / \Delta 11)}$ mutants (fig. S5A-E), and by the fact that such a phenotype is not detectable in any other light receptor mutant available in Platynereis (r-opsin1: fig. S6A,B,E,F,I; c-opsin1: fig. S6C,D,G,H,I, Go-opsin: refs. (26, 27)). 
bioRxiv preprint doi: https://doi.org/10.1101/2021.04.16.439809; this version posted April 18, 2021. The copyright holder for this preprint (which was not certified by peer review) is the author/funder, who has granted bioRxiv a license to display the preprint in perpetuity. It is made available under aCC-BY-NC-ND 4.0 International license.
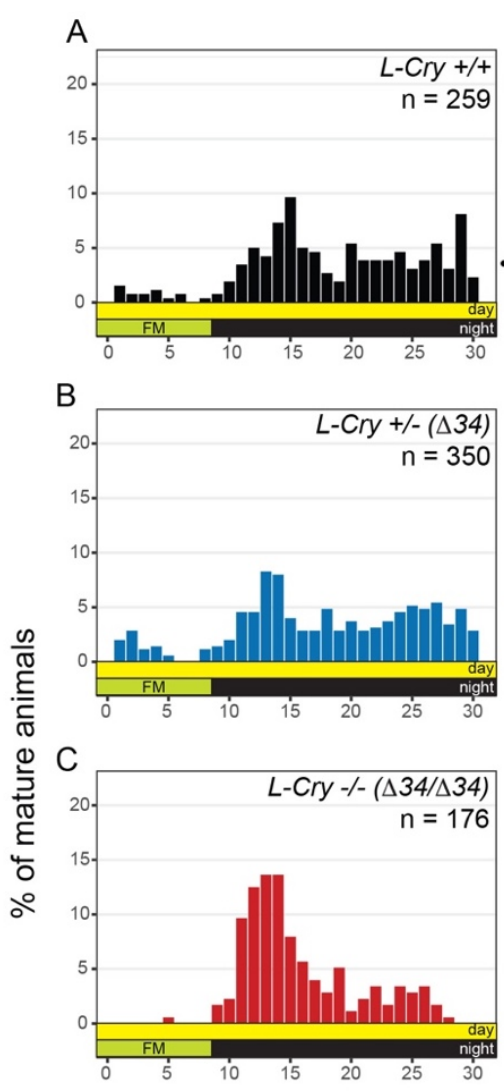

$\mathrm{D}$

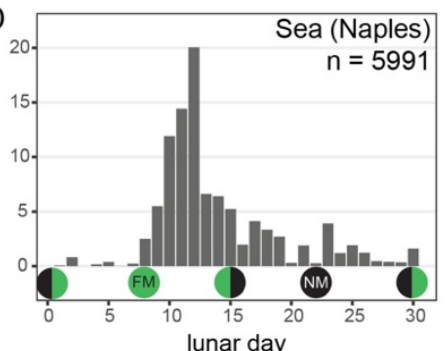

I

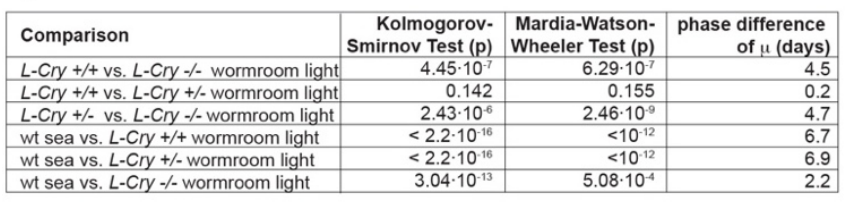

$J$

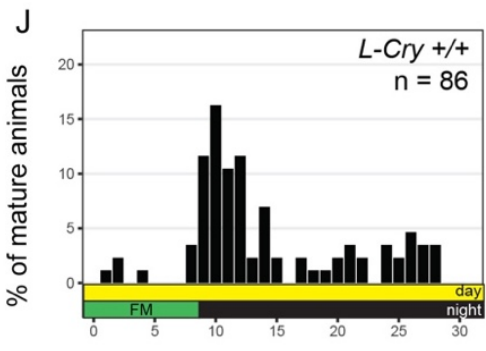

L

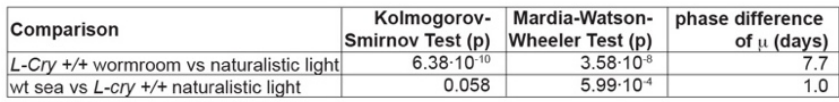

G length of mean $\mu=83.0^{\circ}$

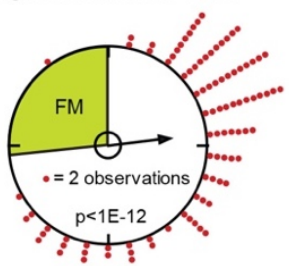

$\mathrm{H}$ mean vector $\mu=56.5^{\circ}$

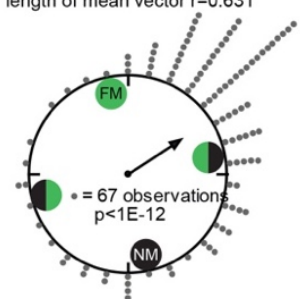

K mean vector $\mu=44.5$ length of mean vector $r=0.398$

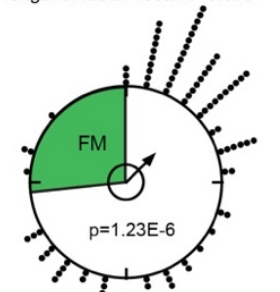
.

$\mathrm{F}$ mean vector $\mu=139.6^{\circ}$
length of mean vector $r=0.222$

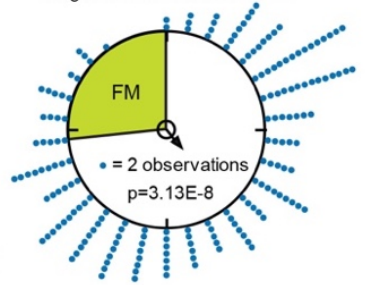

Figure 5: L-Cry shields the circalunar clock from light that is not moonlight

(A-D,J) Spawning of l-cry +/+ (A), l-cry +/$(\Delta 34)$ (B) and l-cry -/-( $\Delta 34 / \Delta 34)$ (C) animals over the lunar month in the lab with 8 nights of moonlight $(A-C)$, under natural conditions in the sea (D, replotted from ref. $(28,29))$ and in the lab using naturalistic sun- and moonlight (J, 8 nights moonlight). (E-H,K) Data as in (A-D,J) as circular plot. $360^{\circ}$ correspond to 30 days of the lunar month. The arrow represents the mean vector $\mu$. $r$ (length of $\mu$ ) indicates phase coherence (measure of population synchrony). p-values inside the plots: Rayleigh Tests. Significance indicates non-random distribution of data points. The inner circle represents the Rayleigh critical value $(p=0.05)$. $(I, L)$ Results of multisample statistics on spawning data shown in $(\mathrm{A}-\mathrm{H}, \mathrm{J}-\mathrm{K})$. 
data from ref. (28)). Given that recent, non-inbred isolates from the same habitat as our lab inbred strains exhibit a very similar (broad) spawning distribution under standard worm culture light conditions (30), we reasoned that the difference in spawning synchrony between the normal wildtype in culture versus at its natural habitat is caused by the rather crude light stimulus typically used for the standard laboratory culture (fig. S3). The high population synchrony of I-cry-/- animals that is close to natural conditions would suggest that L-Cry, as a molecular sun- vs. moonlight discriminator, detects that the highly artificial nocturnal light is not proper moonlight and prevents it from efficiently setting the worms' circalunar clock. This makes the predictions that an extended exposure of the wild-type culture to our "naturalistic" moonlight should lead to a significant increase of entrainment precision.

To test this hypothesis, we assessed the impact of adjusting light intensities and spectrum to naturalistic sun- and moonlight (fig. S3C), maintaining the default temporal regime ( 8 nights of "full moon"). Indeed, just adjusting light qualities indeed improved the precision and phase coherence of population-wide reproduction: After several months under naturalistic sun- and moonlight, wildtype worms spawned with a major peak highly comparable to the wildtype at the natural habitat (Fig. $5 \mathrm{D}, \mathrm{H}$ vs. J,K), and also exhibited an increased population synchrony ( $r=0.398$ compared to $r=0.295$ under standard worm room light conditions). This increased similarity to the spawning distribution at the natural habitat ("Sea") is confirmed by statistical analyses (Fig. 5L), revealing a phase difference of only 1 day, and no significant difference in the Kolmogorov-Smirnov test. In contrast, the spawning distribution of wildtype under standard worm room light versus naturalistic light conditions is highly significantly different in both tests and has a phase difference of 7.7 days (Fig. 5L).

These findings evidence that L-Cry blocks artificial, but not naturalistic moonlight from efficiently resetting the circalunar clock of wildtype animals. This block is removed in I-cry-/- animals, leading to a better synchronization of the I-cry-/- population. This suggests that L-Cry's major role in circalunar clock entrainment could be that of a signal gate-keeper.

\section{l-cry functions as a light signal gate-keeper for circalunar clock} entrainment

A further prediction of this hypothesis is that in a re-entrainment regime using the highly artificial (and not the naturalistic) moonlight, I-cry-/- mutants should entrain better than wild-type.

In fact, we had performed such a re-entrainment experiment early on during the analysis of the I-cry/- mutants. (And were initially puzzled by its results.) We compared the spawning rhythms of I-cry ${ }^{+/+}$ and $\mathrm{I}-\mathrm{cry}^{-/-}$worms under a re-entrainment paradigm, where we provided our bright artificial culture moon at the new moon phase (Fig.6A). Using these highly artificial nocturnal light conditions, the reentrainment of $\mathrm{I}-\mathrm{cry}^{-/-}$animals was both faster and more complete than for their wildtype relatives. This is evident from the linear data analysis and Kolmogorov-Smirnov tests when comparing the 
bioRxiv preprint doi: https://doi.org/10.1101/2021.04 16 439809; this version posted April 18, 2021. The copyright holder for this preprint (which was not certified by peer review) is the author/funder, who has granted bioRxiv a license to display the preprint in perpetuity. It is made available under aCC-BY-NC-ND 4.0 International license.

321 month before the entrainment (month 1) with two months that should be shifted after the 322 entrainment (month D,E) (Fig. 6B,C,F,G). Most notably, while I-cry-/- worms are fully shifted in month 323 E, wildtype animals are mostly still spawning according to the initial lunar phase (boxes, blue 324 arrowheads Fig.6B,C). The faster re-entrainment of $I-c r y^{-1-}$, compared to $I-c r y+/+$ animals is also 325 confirmed by the Mardia- Watson Wheeler test and the phase differences from the circular analysis 326 (Fig. 6D-G). 
bioRxiv preprint doi: https://doi.org/10.1101/2021.04.16.439809; this version posted April 18, 2021. The copyright holder for this preprint (which was not certified by peer review) is the author/funder, who has granted bioRxiv a license to display the preprint in perpetuity. It is made available under aCC-BY-NC-ND 4.0 International license.
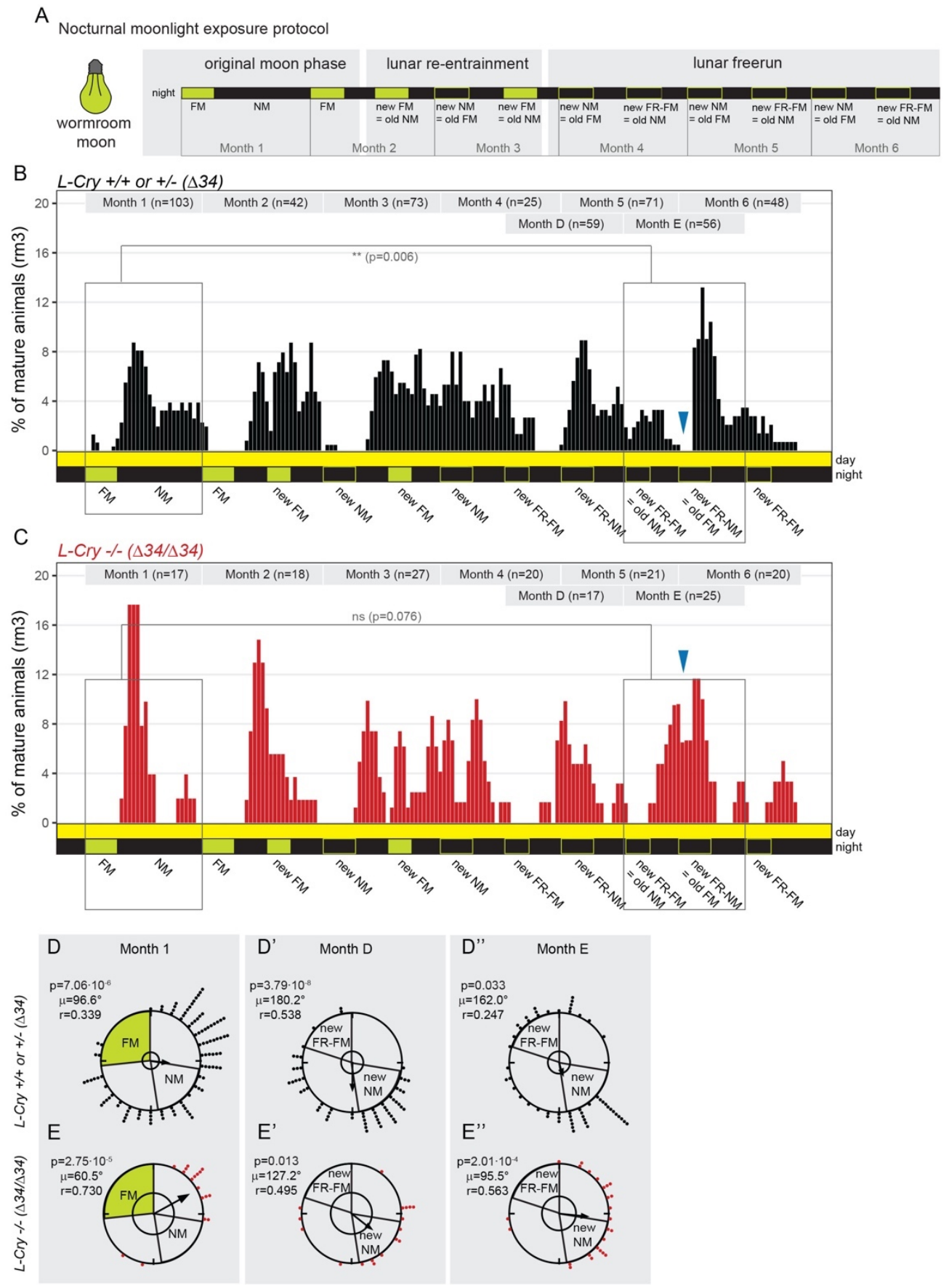

F L-Cry+/+or +/- (434)

$\mathrm{G}_{\text {L-Cry }-(\Delta 34 / \Delta 34)}$

\begin{tabular}{|l|r|r|r|}
\hline Comparison & $\begin{array}{r}\text { Kolmogorov- } \\
\text { Smirnov Test (p) }\end{array}$ & $\begin{array}{r}\text { Mardia-Watson- } \\
\text { Wheeler Test }(p)\end{array}$ & $\begin{array}{r}\text { phase difference } \\
\text { of } \mu \text { (days) }\end{array}$ \\
\hline Month 1 vs D & $1.2 \cdot 10^{-6}$ & $7.5 \cdot 10^{-6}$ & 7.0 \\
\hline Month 1 vs E & 0.006 & 0.002 & 5.5 \\
\hline
\end{tabular}

\begin{tabular}{|l|r|r|r|}
\hline Comparison & $\begin{array}{r}\text { Kolmogorov- } \\
\text { Smirnov Test (p) }\end{array}$ & $\begin{array}{r}\text { Mardia-Watson- } \\
\text { Wheeler Test (p) }\end{array}$ & $\begin{array}{r}\text { phase difference } \\
\text { of } \mu \text { (days) }\end{array}$ \\
\hline Month 1 vs D & 0.002 & 0.002 & 5.6 \\
\hline Month 1 vs E & 0.08 & 0.02 & 2.9 \\
\hline
\end{tabular}

Figure 6: I-cry-/- mutants entrain the circalunar clock faster than wt to a high intensity artificial moonlight stimulus (A) Nocturnal moonlight exposure protocol of lunar phase shift (entrained by 8 nights, phased shifted by 6 nights of artificial culture moon). (B,C) Number of mature animals (percent per month, rolling mean with a window of 3 days) of 1 -cry wildtype (B) and homozygous mutant (C) animals. p-values indicate results of Kolomogorov-Smirnov tests. Blue arrowheads: spawning minima in wt versus mutant populations, respectively. (D-E) Circular plots of the data shown in (B) and (C). Each circle represents one lunar month. Each dot represents one mature worm. The arrow represents the mean vector $\mu$. $r$ (length of $\mu$ ) indicates phase coherence (measure of population synchrony). The inner circle represents the Rayleigh critical value $(p=0.05)$. $(F, G)$ Results of multisample statistics of data in $(D, E)$. 
This provides further evidence that L-Cry normally blocks the "wrong" light from entering into the circalunar clock and thus functions as a gate-keeper to optimize for the correct signal interpretation in the highly complex natural light environment of the worms (Fig.7).

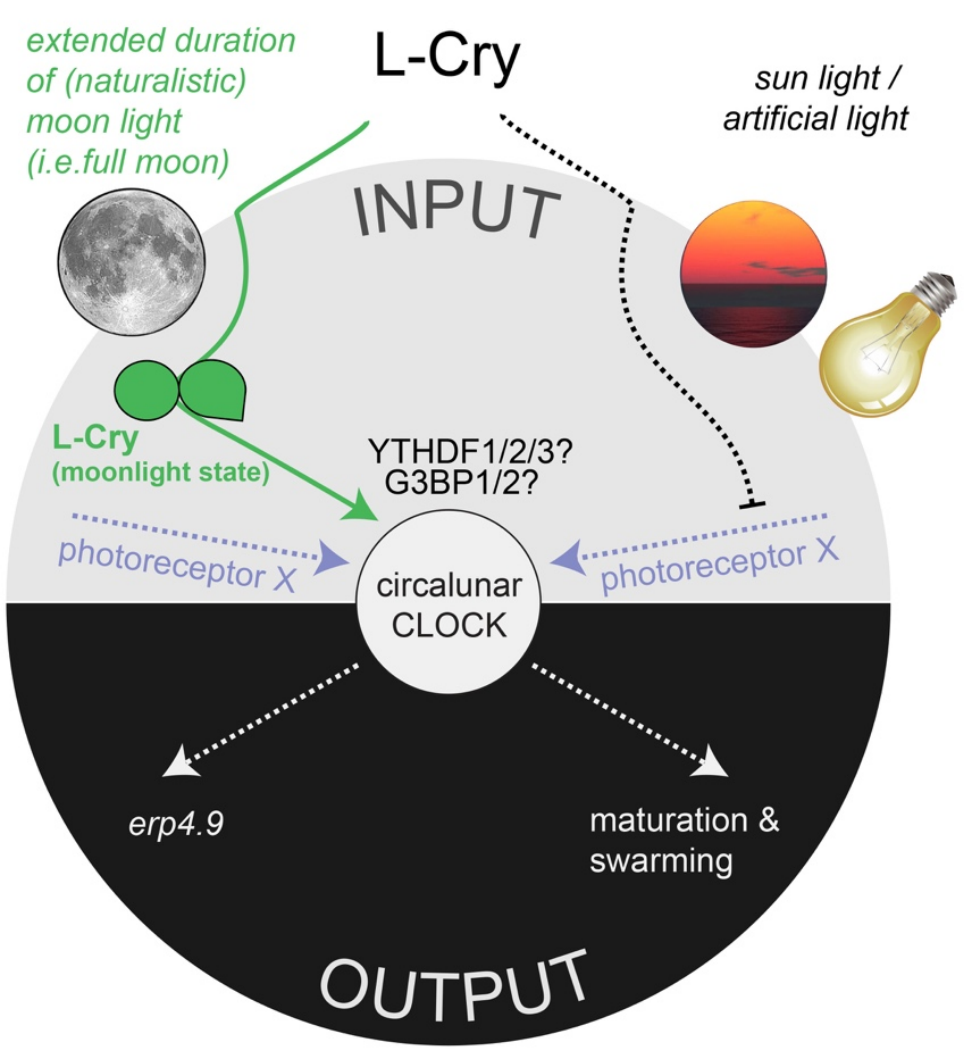

Figure 7. L-Cry functions as moonlight duration and valence detector for circalunar clock entrainment. L-Cry's biochemical property of only reaching the full moonlight state after extended periods of (naturalistic) moonlight illumination allow for a discrimination of moonlight duration. As moon phases are characterized by the duration of the moon on the night sky, moonlight exposure duration translates into moon phase detection. L-Cry also discriminate the valence of light, strongly favoring (naturalistic) moonlight to entrain the circalunar clock. YTHDF1/2/3 and G3BP1/2 are putative L-Cry interactors, possibly mediating part of the signal processes.

\section{L-Cry may functionally couple to two circalunarly regulated proteins}

Finally, in order to next obtain a first understanding how L-Cry might link to the circalunar clock, we employed a biochemical pull-down approach, coupled to mass spectrometry- based identification of candidates.

Purified dark-adapted L-Cry was used as a bait to pull down proteins from wildtype worm head extracts. We identified 11 putative interactors, two of which had been previously identified as circalunarly regulated on protein level (16): a common ortholog of vertebrate Ras-GTPase binding proteins 1 and 2 (hit 2: Pdu- G3BP1/2) and of vertebrate YTH domain containing factors 1, 2 and 3 (hit 5: Pdu- YTHDF1/2/3) (fig. S7A-C). As these interaction candidates could provide insight into the circalunar entrainment pathway, we focused on them. First, we tested if the localization of $g 3 b p 1 / 2$ and $y$ thdf1/2/3 mRNA overlaps with L-Cry positive cells, as a minimal requirement for a biologically relevant interaction. (We tested the mRNA localisation of $g 3 b p 1 / 2$ and $y$ thdf1/2/3 as we at present do not have antibodies against these proteins.) Indeed, g3bp1/2 (Fig. 8A) and ythdf1/2/3 (Fig. 8B) 
bioRxiv preprint doi: https://doi.org/10.1101/2021.04.16.439809; this version posted April 18, 2021. The copyright holder for this preprint (which was not certified by peer review) is the author/funder, who has granted bioRxiv a license to display the preprint in perpetuity. It is made available under aCC-BY-NC-ND 4.0 International license.

showed an overlap in expression domains with I-cry (compare Fig. 2D) in the posterior oval-shaped domain and the eyes. We next combined in situ hybridisation with L-Cry antibody staining. Indeed, both $g 3 b p 1 / 2$ and $y$ thdf $1 / 2 / 3$ localize to L-Cry positive cells as indicated by the dark precipitate surrounding an L-Cry+ nucleus (arrowheads pointing at examples in Fig. 8 $\mathrm{A}^{\prime}, \mathrm{B}^{\prime}$ ), supporting that they are present in the same cells and can thus in principle function together with L-Cry.
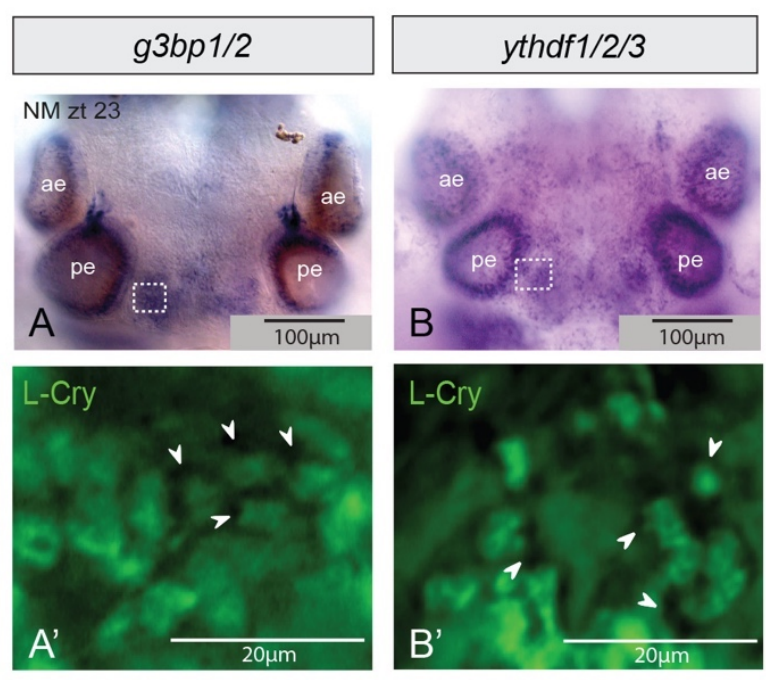

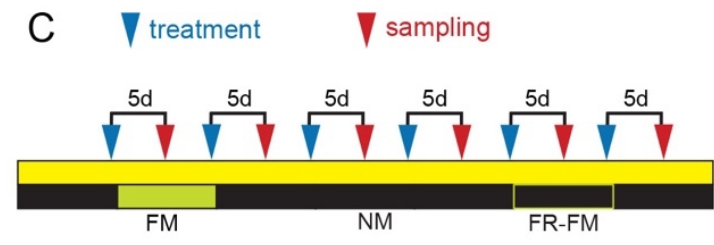

D

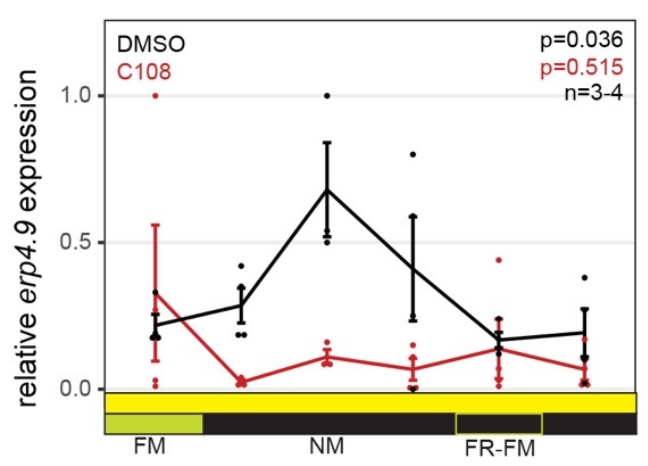

$\mathrm{F}$

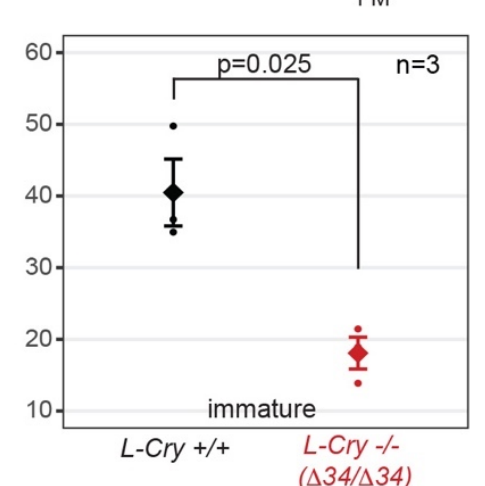

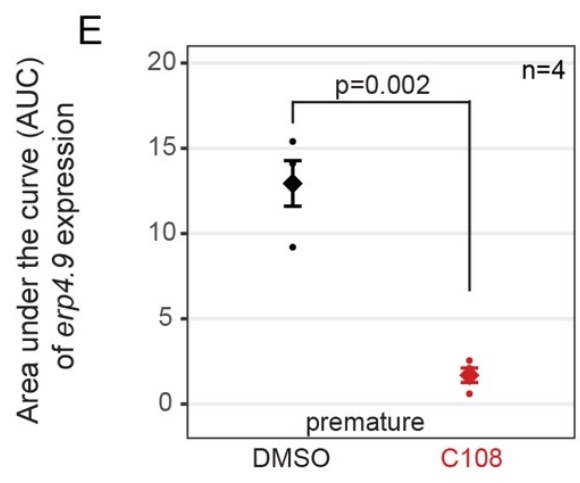

Figure 8 : The putative L-Cry interactors G3BP1/2 and YTH1/2/3 are co-expressed with L-Cry and pharmacological inhibition of G3BP1/2 affects the circalunar clock.

(A,B) Whole-mount in situ hybridization (WMISH) in premature worm heads combined with L-Cry antibody staining (green). Note that the purple precipitate of the WMISH signal is seen black on the fluorescent images. Arrowheads: cells with cytoplasmic WMISH signal and nuclear L-Cry. Rectangles in $(A)$ and $(B)$ indicate region shown in $\left(A^{\prime}, B^{\prime}\right)$. ae, anterior eye; pe, posterior eye. (C) Experimental scheme of C108 treatment. (D) Expression levels of erp4.9 over the lunar month upon C108 treatment in heads of premature animals. p-values: one-way-ANOVA. (E) Area under the curve of erp4.9 expression shown in (D). (F) Area under the curve of erp4.9 expression in immature worm heads calculated from the non-normalized data from Fig. $4 \mathrm{H}$. p-values in (E) and (F) are results of t-tests. Individual data points as well as mean \pm SEM are shown in (D-F). Note that different stages of animals were used in (E) versus (F), impacting on absolute levels (25), but not relative regulation.

If either of the two putative functional L-Cry partners is indeed involved in the circalunar mechanism, interfering with them should have an effect on circalunar timing. We thus treated pre-mature worms with the G3BP2 inhibitor C108 (31) throughout a circalunar month (scheme Fig.8C) and measured erp4.9 expression levels as a marker for circalunar clock phase (Fig. 4H). Upon a test of multiple concentrations for short periods (data not shown), we decided to perform this multi-week 
experiment with $10 \mu \mathrm{M}$ C108. This treatment caused erp4.9 expression to no longer show significant changes in expression over the lunar month (Fig.8D) and furthermore reduced overall erp4.9 expression levels (Fig. 8E). This indicated that erp4.9 circalunar transcript rhythmicity is controlled by Pdu-G3BP1/2. In comparison, g3bp1/2 expression levels were not affected by C108 treatment (fig. S7D,E), indicating that the effect on erp4.9 is not caused by an overall effect on transcription. It is noteworthy that erp4.9 levels were also significantly reduced in I-cry-/- worms (Fig. 8F). These data could indicate a possible involvement of G3BP1/2 in the L-Cry signalling of different light valences to the circalunar clock (Fig. 7).

\section{Discussion}

Our work delivers the first molecular entry point into the mechanisms underlying a moonlightentrained monthly oscillator. It provides strong evidence that L-Cry is part of the lunar entrainment pathway and suggests a link to proteins that are themselves circalunarly regulated (Fig. 7). By this we advance the existing knowledge on Cryptochrome functions and non-circadian oscillators at multiple levels.

First, we provide biochemical evidence that at dim light levels, corresponding to moonlight in nature, L-Cry can accumulate photons over time. L-Cry's photoreduction response to this accumulation is in its duration markedly different from its rapid, well-established response to strong light and nonlinear, suggesting that a different "moonlight" signalling state might exist. Second, consistent with different L-Cry states under dim and strong light, L-Cry under naturalistic moonlight is not following the conventional cytoplasmic degradation pathway, but localizes at higher levels to the nucleus. This suggests that different cellular compartments convey the different light messages to different downstream pathways. The regulation of the subcellular localization depending on light regime prompted us to look for any conventional nuclear localization sequences, which we could not detect in L-Cry.

Upon exposure to naturalistic daylight, L-Cry rapidly moves to the cytoplasm, where its protein levels become reduced, fully consistent with our previous data in S2 cells (14). In Drosophila melanogaster $\mathrm{dCry}$ is degraded via its light-induced interaction with the circadian clock protein Timeless and subsequent JETLAG (JET) ubiquitin-ligase-mediated proteolytic degradation (32). Given that both Timeless and Jetlag exist in Platynereis (Pdu-Tim: (14), Pdu-Jet: reciprocal best blast hit in ESTs), it is possible that a similar mechanism exists in Platynereis. It is however noteworthy that timeless transcript levels are regulated by room_light in the bristle worm (14), indicative of additional levels of regulatory complexity. 
In contrast to the suggested mechanism about L-Cry's fate in the cytoplasm based on existing knowledge from its Drosophila ortholog, the signalling downstream of nuclear L-Cry is at present completely enigmatic. It is clear from our data (and the accompanying manuscript Zurl et al) that neither darkness nor naturalistic moonlight causes degradation of nuclear L-Cry. This might indicate that ratios of cytoplasmic versus nuclear L-Cry could be important for circalunar clock moonlight entrainment. To complicate matters even further- it is possible that different spatial expression domains, such as eye versus brain, need to be considered separately in their responses to extended naturalistic moonlight and subsequent downstream signalling cascades.

Without at present being able to provide spatial resolution, we offer a first glimpse at possible downstream players, the proteins G3BP1/2 and YTHDF1/2/3. The localization of the mRNA of both factors to L-Cry positive cells at least allows for a function of these proteins together in the same cells. However, it is absolutely clear that follow-up studies with antibodies against both proteins will be required to further verify and understand their interactions with L-Cry, and their possible connection to the circalunar clock. The fact that a treatment of worms with the G3BP2 inhibitor C108 results in a disruption of the circalunar oscillations of erp4.9 provides support for placing it in a pathway with L-Cry and opens the question about possible functions in connection with the circalunar clock. Interestingly, a common feature of the orthologs of both G3BP1/2 and YTHDF1/2/3 are their function in RNA-binding and regulation(33-35). Furthermore, a screen for interacting proteins of YTHDF1, 2 and 3 in mammalian cells revealed G3BP1 and 2 among the top 25 interacting proteins for each YTHDF(35), suggesting that these proteins can function within a complex. In the perspective of possible roles in mRNA translation and stability this could provide an explanation for our previous observation that changes on protein rather than transcript level are prominent between the NM and FR-FM phase of the worm's circalunar clock (25), an observation that had already prompted us to speculate about post-transcriptional processes being of high importance to the functionality of the circalunar oscillator. But how does this connect with the localization of L-Cry to the nucleus under darkness and - even more- under moonlight? Could it be possible that moonlight removes remaining L-Cry from G3BP1/2 and YTHDF1/2/3 containing complexes in the cytoplasm and by this impacts on the regulation of mRNA translation and stability? Is the observation that more cells possess only nuclear L-Cry after 7 nights of naturalistic full moon compared with more nuclear and cytoplasmic L-Cry under dark NM nights potentially relevant in this context? To further complicate matters- it is in our opinion highly likely that L-Cry also has a function in the nucleus itself. This could be independent of G3BP1/2 and YTHDF1/2/3, like transcriptional regulation. This has been shown to occur for its ortholog in Drosophila (36). However, also G3BP1/2 and YTHDF1/2/3 orthologs can be present in the nucleus $(33,37)$, and G3BP1/2 possesses a highly conserved NTF2-like domain. 
transport (38). It is thus also possible that the putative interaction between G3BP1/2 and L-Cry is involved in the cytoplasmic/nuclear shuttling of L-Cry and/or nuclear functions.

Connected to the question of the transmission of the moonlight signal to the circalunar oscillator is also a better understanding of L-Cry's "moonlight state". Is this state "just" a partial photoreduction of the state reached upon artificial sunlight exposure or perhaps also a conformationally different state with distinct formation and decay kinetics? And what is the role of the L-Cry dimers? An intriguing observation is, that in presence of moonlight the moonlight state can be stably maintained over several hours, whereas the sunlight state completely reverts to the fully-oxidized dark-state within minutes without accumulating the moonlight state while transitioning through partial photoreduction (Fig.1J). These different responses to moonlight illumination suggest that the moonlight- and sunlight states are conformationally and kinetically not equivalent (fig. S2I). Based on its sequence homology and the similarity of its FAD photoreaction to Drosophila CRY (dCry), it is conceivable that L-Cry also displaces the regulatory C-terminal tail in the photoreduced state as observed for dCry (21)'(39). However, as dCry is monomeric, L-Cry homodimer formation may impact these conformational changes, and these may further vary depending on whether moonlight or sunlight operates on the initial dark-state L-Cry homodimer. We propose, that partial FAD photoreduction in the moonlight state could be related to the formation of asymmetric L-Cry dimers, where one monomer retains oxidized FAD, while in the second monomer FAD is photoreduced to $F A D^{\circ-}$ (Fig. 1J). This requires, that the flavins in the two L-Cry monomers have different redox potentials, likely resulting from different chemical environments due to conformational differences between the monomers (Fig. S2I). Hence different amounts of energy (photon numbers) would be needed to photoreduce the flavins in the two L-CRY monomers. Moonlight, due to its very low intensity can only induce the lower energy transition, resulting in the partially photoreduced moonlight state. In presence of intense sunlight, however, the larger energy barrier to photoreduce the second flavin can also be overcome. Certainly, more extensive mechanistic studies are required to further support our model. However, this model is consistent with all our current in vitro data, and moreover, it plausibly illustrates how the very different intensities of moon- and sunlight can lead to the formation of conformationally distinct dark state (new moon), moonlight state (full moon) and sunlight state L-Cry proteins. Thereby L-Cry could translate different light qualities into different cellular signaling events, e.g. by changing L-Cry's subcellular localizations and cellular degradation rates (Fig. 3), to ultimately affect moonlight dependent physiology (Fig. 4-7).

Finally, an evolutionary consideration: Monthly synchronization by the moon has been documented for a wide range of organisms- including brown and green algae, corals, crustaceans, worms, but also vertebrates (reviewed in (6)). Furthermore, recent reports also provide increasing evidence that the 
lunar cycle influences human behaviour (reviewed in $(40,41)$ ). Are the lunar effects mediated by conserved or different mechanisms? It is important to take the period lengths into consideration. In other words- just because an oscillation is controlled by the moon, doesn't mean it has the same mechanism. There is a big difference between a period length in the range of hours, versus a period length in the range of weeks. Thus, also for evolutionary comparisons, tidal and circalunidian/circadian rhythms should be considered differently from monthly rhythms (also see accompanying manuscript Zurl et al.).

When considering monthly oscillators with period lengths in the range of weeks, our implication of LCry as a light receptor in the circalunar entrainment pathway at first glance rather suggests that such monthly oscillator might not be conserved, given that direct L-Cry orthologs are not present in all the groups that are affected by the lunar cycle (42). However, taking further aspects into account, such a conclusion might be too quick. Could other members of the Cry/photolyase family take over similar functions? Could it also be that other cues, such as changes in the earth magnetic field provide additional entrainment cues? Furthermore, our entrainment data suggest the presence of additional moonlight photoreceptors.

Last, but not least the molecular mechanisms underlying the circalunar oscillator also await identification, and it is possible that conservation exists on this level. Examples are known from circadian biology and it will now require further work to reach a similar level of understanding for moon-controlled monthly rhythms and clocks.

\section{Materials and Methods}

\section{Natural light measurements}

Under water measurements of natural light at the habitat of Platynereis dumerilii were acquired using a RAMSES-ACC-VIS hyperspectral radiometer (TriOS $\mathrm{GmbH}$ ) for UV to IR spectral range. In coastal waters of the Island of Ischia, in the Gulf of Naples, the two radiometers were placed on sand flat at $5 \mathrm{~m}$ depth near to Posidonia oceanica meadows, which are a natural habitat for $P$. dumerilii. Measurements were recorded automatically every $15 \mathrm{~min}$ across several weeks in the winter 2011/2012. To obtain a fullmoon spectrum, measurements taken from 10pm to 1am on a clear fullmoon night on the 10.11.2011 were averaged. To subtract baseline noise from this measurement, a NM spectrum was obtained by averaging measurements between 7:15pm to $5 \mathrm{am}$ on a NM night on 24.11.2011, and subtracted from the FM spectrum. Resulting spectrum: fig. S3A. To benchmark these moonlight spectra measured under water with moonlight measured on land, we compared the underwater spectra to a publicly available full moon spectrum measured on land on 14.04.2014 in the Netherlands (fig. S3G, spectrum available at http://www.olino.org/blog/us/articles/2015/10/05/spectrum-of-moon-light). As expected, light with 
longer wavelengths was strongly reduced in the underwater measurements compared to the surface spectrum, since longer wavelengths penetrate water less efficiently. For the sunlight spectrum, measurements taken from 8am to 4pm on a sunny day on 9.11.2011 were averaged.

\section{Naturalistic Light Systems (NELIS devices)}

To emulate naturalistic sunlight and moonlight conditions, we employed NELIS (Natural Environment Light Intensity System) (Marine Breeding Systems $\mathrm{GmbH}$ )(22). The naturalistic moonlight device was composed of a combination of LEDs and an Ulbricht sphere for homogenous light mixing. For improved light distribution across the shelf, a naturalistic moonlight device was attached to each end of an acrylglass rod (two light sources and one rod per shelf). For details on the naturalistic sunlight source see ref. (22). Light spectra were measured using ILT950 Spectroradiometer (International Light Technologies).

\section{Cloning and recombinant virus generation for L-Cry}

Full length N-terminally His6-tagged Platynereis dumerilii L-Cry (1-567) was heterologously expressed in Spodoptera frugiperda (Sfg) insect cells using the Bac-to-Bac baculovirus expression system with the pCoofy27 expression vector. $1 * 10^{6}$ Sfg cells were transfected with recombinant bacmid DNA using Cellfectin. The first generation PO virus was harvested 3 days after bacmid transfection. A further virus amplification step was carried out and the P1 virus stock was used for protein expression. The volume of P1 virus stock to be added for sufficient protein expression was determined by test expression.

\section{Protein expression and purification}

Sfg cells were grown as suspension cultures in sf900II media at $27^{\circ} \mathrm{C}, 80 \mathrm{RPM} .1 \mathrm{~L}$ of $1 * 10^{6} \mathrm{Sfg}$ cells $/ \mathrm{ml}$ were transfected with $\mathrm{P} 1$ virus stock and incubated at $27^{\circ} \mathrm{C}$ for $72 \mathrm{~h}$. Cells were harvested by centrifugation at $7000 \mathrm{rpm}$ for $20 \mathrm{~min}$ and stored at $-80^{\circ} \mathrm{C}$ until purification. All purification steps were carried out in dark or dim red light conditions. Columns were wrapped with aluminum foil to avoid light-activation of L-Cry. The cell pellets were resuspended in lysis buffer ( $20 \mathrm{mM}$ Tris pH 7.5, $150 \mathrm{mM} \mathrm{NaCl}, 20 \mathrm{mM}$ imidazole, $5 \%$ glycerol, $5 \mathrm{mM} \beta$-mercaptoethanol) and lysed using a microfluidizer. The lysate was centrifuged at $27000 \mathrm{rpm}$ for $45 \mathrm{~min}$ and the clarified supernatant incubated with nickel beads for $1 \mathrm{~h}$. The nickel beads were loaded onto a batch column, washed with 50-100 mM imidazol and the L-Cry protein was eluted with $250 \mathrm{mM}$ imidazole. Elution fractions containing L-Cry were concentrated, diluted with low salt buffer $(50 \mathrm{mM}$ Tris pH 7.5, $50 \mathrm{mM} \mathrm{NaCl}, 5 \%$ glycerol, $1 \mathrm{mM}$ DTT) and loaded onto a $5 \mathrm{ml}$ Hitrap $\mathrm{Q}$ sepharose anion exchange column (GE Healthcare). A gradient from $0 \%$ to $100 \%$ high salt buffer ( $50 \mathrm{mM}$ Tris pH 7.5, $1 \mathrm{M} \mathrm{NaCl}, 5 \%$ glycerol, $1 \mathrm{mM}$ DTT) was applied. L-Cry containing fractions were pooled, concentrated and loaded onto a HiLoad S200 16/60 size exclusion chromatography (SEC) column (buffer 25 mM Bis-Tris propane pH 
8.0, $150 \mathrm{mM} \mathrm{NaCl}, 5 \%$ glycerol, $1 \mathrm{mM}$ TCEP). Fractions containing pure L-Cry were pooled, concentrated to $10 \mathrm{mg} / \mathrm{ml}$ and snap frozen in liquid nitrogen for storage at $-80^{\circ} \mathrm{C} .2 \mathrm{mg}$ of L-Cry was obtained from $10 \mathrm{~g}$ of pellet. The identity of the L-Cry protein was confirmed by mass spectrometry.

550 Flavin Mononucleotide (FMN), Flavin Adenine Dinucleotide (FAD) and Methenyltetrahydrofolate 551 (MTHF) were dissolved in buffer (25 mM Bis-Tris propane $\mathrm{pH} 8.0,150 \mathrm{mM} \mathrm{NaCl}, 5 \%$ glycerol) and run at $1 \mathrm{ml} / \mathrm{min}\left(20^{\circ} \mathrm{C}\right.$ ) over a Macherey-Nagel C18 Gravity-SB $(150 / 4 / 5 \mu \mathrm{m})$ column to separate the chromophores by reverse phase (RP) HPLC analyses. A gradient from $20-100 \%$ of methanol against water $(+0.1 \%$ Trifluoroacetic acid) was used for optimal separation. To analyse the chromophore content of L-Cry, purified L-Cry was heat-denatured for $5 \mathrm{~min}$ at $97^{\circ} \mathrm{C}$ and centrifuged at $14000 \mathrm{RPM}$ for $10 \mathrm{~min}$ at $4^{\circ} \mathrm{C}$. The supernatant was subjected to RP-HPLC analysis. The chromophores were monitored by absorption at $370 \mathrm{~nm}$.

Analytical Size Exclusion Chromatography (SEC) and SEC coupled with Multiangle light scattering (SEC-MALS)

560 Analytical SEC of dark-state L-Cry was carried out on a S200 10/300 size exclusion column (SEC buffer $56125 \mathrm{mM}$ Bis-Tris propane $\mathrm{pH}$ 8.0, $150 \mathrm{mM} \mathrm{NaCl}$ ) under red light conditions. SEC-MALS was carried out 562 to determine the exact molecular weight and oligomeric state of purified L-Cry based on the SEC 563 elution volume and light scattering. For SEC-MALS, purified L-Cry was loaded onto a Superose 6 564 10/300 size exclusion column and run at a flowrate of $0.4 \mathrm{ml} / \mathrm{min}$ in SEC buffer. MALS data were obtained from a DAWN DSP instrument (Wyatt Tech, Germany) and processed using ASTRA 4.90.07. Elution volumes and corresponding molecular weight of calibration standards: $10.3 \mathrm{ml}-670 \mathrm{kDa}$ (Thyroglobulin), $13.67 \mathrm{ml}-158 \mathrm{kDa}$ ( -globulin), $15.71 \mathrm{ml}-44 \mathrm{kDa}$ (ovalbumin), $17.42 \mathrm{ml}-17 \mathrm{kDa}$ (myoglobin) and $20.11 \mathrm{ml}-1350 \mathrm{Da}$ (vitamin B12).

UV/VIS spectroscopy on L-Cry: Blue light-, sunlight- and moonlight photoreduction and dark recovery

571 UV/Visible absorption spectra of the purified L-Cry protein were recorded on a Tecan Spark 20M 572 plate reader unless otherwise stated. A light-state spectrum of L-Cry with fully photoreduced FAD ${ }^{\circ-}$ 573 was collected after illuminating dark-adapted L-Cry for $110 \mathrm{sec}$ with a $450 \mathrm{~nm}$ blue light emitting 574 diode (fig S3D,E; $6.21 \times 10^{16}$ photons $/ \mathrm{cm}^{2} / \mathrm{sec}$ at the sample). To analyze sunlight- and moonlight 575 dependent FAD photoreduction, dark-adapted L-Cry (kept on ice) was continuously illuminated with 576 naturalistic sunlight (fig. S3C,E; $1.55 \times 10^{15}$ photons $/ \mathrm{cm}^{2} / \mathrm{sec}$ at the sample) or naturalistic moonlight 577 (fig. S3C,E; $9.65 \times 10^{10}$ photons $/ \mathrm{cm}^{2} / \mathrm{sec}$ at the sample) and UV-VIS spectra (300 - $700 \mathrm{~nm}$ ) were collected at different time points. 
Dark recovery kinetics (FAD reoxidation) of L-Cry at $18^{\circ} \mathrm{C}$ following illumination with blue-light (110 sec), sunlight ( $20 \mathrm{~min}$ on ice) or moonlight ( $6 \mathrm{~h}$ on ice) were measured by recording absorbance changes at $450 \mathrm{~nm}$ over time or by extracting $450 \mathrm{~nm}$ absorbance values from complete UV/VIS spectra. To measure dark recovery kinetics on ice, complete UV-VIS spectra (300 - $700 \mathrm{~nm}$ ) were collected at different time points following $110 \mathrm{sec}$ blue light- or 20 min sunlight illumination and absorbance values at $450 \mathrm{~nm}$ were extracted from the full spectra (sample was kept on ice and in darkness between measurements). Additionally, a temperature controlled Jasco V-550 UV-VIS spectrophotometer was used to determine dark recovery kinetics of L-Cry (after 110 sec blue-light) at $6^{\circ} \mathrm{C}$ based on absorbance changes at $450 \mathrm{~nm}$. The time constants for dark recovery were calculated by fitting a single exponential curve to the experimental data. Spectra were analyzed using Origin (Version 7.5/10.5(trial); OriginLab Corporation, Northampton, MA, USA).

\section{Recovery of L-Cry dark state in presence of moonlight}

To assess if moonlight can maintain the light state, L-Cry was initially illuminated with sunlight for 20 min or with blue light for $110 \mathrm{sec}$, followed by continuous moonlight illumination up to 6 hours with the sample kept on ice. Complete UV-VIS spectra $(300-700 \mathrm{~nm})$ were collected at different time points. Absorbance values at $450 \mathrm{~nm}$ were taken from the complete spectra obtained between $5 \mathrm{~min}$ and $2 \mathrm{~h} 30 \mathrm{~min}$ moonlight exposure and used to determine the time constant for recovery of oxidized FAD after blue-light or sunlight induced photoreduction in presence of moonlight.

\section{Sunlight illumination of moonlight activated L-Cry}

To assess if sunlight can further increase FAD photoreduction starting from the moonlight activated state, L-CRY was first illuminated with continuous moonlight for 6 hours, followed by 20 min of sunlight illumination (on ice). Complete UV-VIS spectra from $300-700 \mathrm{~nm}$ were measured in each case.

\section{Pull-down of L-Cry interactors and mass spectrometry}

Pre-purified L-Cry with $\mathrm{N}$-terminal $\mathrm{His}_{6}$ tag was bound to magnetic nickel beads. 20 frozen worm heads (ZT20) from new moon phase were resuspended in buffer (50mM Tris pH 7.5, $150 \mathrm{mM} \mathrm{NaCl}$, $2.5 \mathrm{mM} \mathrm{MgCl}_{2}, 1 \%$ Triton $\mathrm{X}, 1 \mathrm{mM}$ phenylmethylsulfonyl fluoride, benzonase and cOmplete ${ }^{\text {TM }}$ EDTAfree protease inhibitor cocktail tablets) and lysed by sonication. The tubes were wrapped with aluminum foil and the pull-down experiment was done under red light. L-Cry bound to magnetic nickel beads was used as bait (sample) and empty nickel beads as control. The worm head lysate was incubated with magnetic nickel beads (sample and control) for $3 \mathrm{~h}$ at $4^{\circ} \mathrm{C}$ on rotation. The beads were washed thrice with resuspension buffer (without $1 \%$ Triton $\mathrm{X}$ ) and finally eluted with Lithium Dodecyl Sulfate (LDS) buffer at $70^{\circ} \mathrm{C}$ for $10 \mathrm{~min}$. The samples were loaded on NuPAGE $4-12 \%$ Bis-Tris commercial gels, excised and prepared for in-gel digestion and dimethyl labelling. The experiment 
613

614

615

616

617

618

619

620

621

622

623

624

625

626

627

628

629

630

631

632

633

634

635

636

637

638

639

640

641

642

\begin{tabular}{|l|l|}
\hline Primer & Sequence 5'-3' \\
\hline I-cry_fwd & AAGAGAAGACTGACGATTGGGAC \\
\hline I-cry_rev & CTGCAACTTCCCCATCCC \\
\hline
\end{tabular}

Primers used for l-cry genotyping

643 Full length I-cry cDNA- GenBank: MW161054

\section{Worm Culture}

\section{Generation and Genotyping of $l$-cry KO worms} (168bp: wildtype, $134 \mathrm{bp}: \Delta 34+\Delta 9$ mutant allele, $157 \mathrm{bp}: \Delta 11$ mutant allele).

was done in 2 replicates. Each sample and control elution was then used for labelling the digested peptides with a heavy and light label (forward experiment) and with switching the label (reverse experiment). The light and heavy labelling provided a list of interactors specifically enriched with LCry as bait as compared to the control. The digested and purified peptides were later analyzed by mass spectrometry. Q Exactive Plus mass spectrometer (Thermo Scientific) was used for all mass spectrometry experiments. Stable-isotope dimethyl labeling was used for quantitative proteomics. The MaxQuant software package (Version 1.5.2.8) was used for data analysis (43-45) with the Uniport database Platynereis_dumerilli_(CLAMWORM)_Uniport_20190116.fasta and the in-house generated worm head database Pdu_HeadRef_Prot_v4.fasta (25). Data analysis was done by MaxQuant (Version 1.5.2.8) with default settings, except re-quantify was activated against an inhouse generated worm head database Pdu_HeadRef_Prot_v4.fasta. The output was further filtered by removing known contaminants, reverse data base binders and proteins only identified by site modification. We also filtered for minimum 2 peptides (minimum 1 unique) per protein group.

Platynereis dumerilii were grown as previously described $(14,46)$. All animal work was conducted according to Austrian and European guidelines for animal research. Photoperiod 16:8 (L/D), circalunar entrainment: nocturnal light for 6-8 nights (see Figure legends for each experiment) every 29 to 30 days (centering around full moon ("inphase") or new moon ("outphase") in Vienna). Light spectra and intensities of fig. S3 were measured with a recently calibrated ILT950 spectrometer (International Light Technologies Inc Peabody, USA) and converted to photons $/ \mathrm{cm}^{2} / \mathrm{s}$.

Design and construction of TALENs targeting l-cry is described in (23). For genotyping, DNA extraction of immature and premature worms was conducted by cutting 5-10 tail segments with a scalpel and incubating them in $20 \mu \mathrm{l} 50 \mathrm{mM} \mathrm{NaOH}$ at $95^{\circ}$ for $20 \mathrm{~min}$. After adding $5 \mu \mathrm{l}$ of $\mathrm{Tris} / \mathrm{HCl} \mathrm{pH} 7.5$, the supernatant was used as template for the PCR reaction. Mature worms were frozen as whole at $20^{\circ} \mathrm{C}$ and DNA was later extracted using NucleoSpin Tissue Mini kit for DNA from cells and tissue (Macherey-Nagel). PCR was performed with OneTaq Quick-Load 2x Master Mix with Standard Buffer (New England Biolabs). PCR product was run on an agarose gel and genotype was determined on size 


\section{Monoclonal Antibody Production}

645 A peptide consisting of amino acids 52 - 290 of L-Cry protein (GenBank ID: MT656570, predicted size $64625 \mathrm{kDa}$ ) was cloned and expressed in bacteria cells. Subsequently, this peptide was purified and used

647 for mouse immunization, thereby acting as epitope in production of a monoclonal antibody against L648 Cry. Upon screening of multiple clones, two clones (4D4-3E12-E7 and 5E3-3E6-E8) were selected and 649 used in combination. Monoclonal antibodies were produced by and purchased from the Monoclonal 650 Antibody Facility at Max Perutz Labs (Medical University of Vienna, Department of Medical 651 Biochemistry).

\section{Immunohistochemistry, microscopy and L-Cry localization determination}

653 Worm heads were dissected with jaws and fixed in $4 \%$ PFA for $24 \mathrm{~h}$ at $4^{\circ} \mathrm{C}$. Samples were 654 subsequently permeabilized using methanol, digested for $5 \mathrm{~min}$ with Proteinase $\mathrm{K}$ at room 655 temperature without shaking and post-fixed with 4\% PFA for $20 \mathrm{~min}$ at room temperature. Next, 656 samples were washed 5 times for 5 min with $1 \times$ PTW and incubated in hybridization mixture(47) used 657 in in situ hybridization protocol, at $65^{\circ} \mathrm{C}$ overnight. Worm heads were washed with $50 \%$ 658 formamide/2X SSCT - standard saline citrate containing 0.1\% Tween $20^{\circledR}$ (Sigma Aldrich) (2x, 20 min), 659 then with 2 X SSCT $(2 x, 10 \mathrm{~min})$ and with $0.2 \mathrm{XSCT}(2 \mathrm{x}, 20 \mathrm{~min})$; all washing steps at $65^{\circ} \mathrm{C}$. After 660 blocking for $90 \mathrm{~min}$ with $5 \%$ sheep serum (Sigma-Aldrich) at room temperature, samples were incubated in L-Cry antibodies 5E3-3E6-E8 (1:100) and 4D4-3E12-E7 (1:50) in 5\% sheep serum (SigmaAldrich). Secondary antibody, Cy3 goat anti-mouse IgG (A10521, Thermo Fisher Scientific) was diluted $1: 400$ in $2.5 \%$ sheep serum (Sigma-Aldrich). Incubations were done for at least $36 \mathrm{~h}$ at $4^{\circ} \mathrm{C}$ shaking and after each incubation time, samples were washed with 1x PTW three times for $15 \mathrm{~min}$ at room temperature and a fourth time over night at $4^{\circ} \mathrm{C}$. After this, Höchst 33342 (H3570, Thermo Fisher Scientific), diluted 1:2000, was added for at least $30 \mathrm{~min}$ at room temperature. Samples were then washed three times for 15 min with 1 PTW at room temperature and mounted with $25 \mathrm{mg} / \mathrm{ml}$ DABCO (Roth/Lactan) in an $87 \%$ glycerol (Sigma-Aldrich) solution. All solutions were made with $1 \mathrm{x}$ PTW (PBS $+0.1 \%$ Tween 20 ${ }^{\circledR}$ ) (Sigma Aldrich). Heads were imaged on a Zeiss LSM 700 laser scanning confocal microscope using LD LCI Plan-Apochromat 25X, Plan-Apochromat 40X by CHD: T-PMT detection system and Zeiss ZEN 2012 software. Lasers: DAPI $405 \mathrm{~nm}$ and Cy3 $555 \mathrm{~nm}$.

672 Categorical scoring: Using Fiji/ImageJ (48), nuclear outlines were marked as Regions Of Interest (ROI) 673 on the $405 \mathrm{~nm}$ channel images (Höchst staining). ROls were then used for scoring of the signal 674 localization (inside $=$ nucleus versus outside $=$ cytoplasm) on the $555 \mathrm{~nm}$ channel of the same images 675 (L-Cry). 
678 Interest (ROI). L-Cry signal was then determined for these nuclear ROls using in Fiji/lmageJ (48).

679 Signal intensity was determined by calculating Corrected Total Cell Fluorescence (CTCF) using the 680 formula CTCF=Area (ROI_1 )*Mean (ROI_1 )-Area (ROI_1)*Mean(ROI_(background ROIs)). A sum of 681 CTCF values of all the nuclei was subtracted from the CTCF value of the whole brain area, to obtain the corresponding value for non-nuclear, i.e. cytoplasmic signal. Finally, the ratio between nuclear and non-nuclear (cytoplasmic) signal intensity was calculated for corresponding regions of different worm heads to compare between different ZTs.

685

686

687

688

689

690

691

692

693

694

695

696

697

698

699

700

701

702

703

704

705

706

707

708

709

710

711

\section{Protein extraction and Western Blots}

Per biological replicate four premature worms were anaesthetized (7.5\% $\mathrm{MgCl}_{2}-/ \mathrm{H}_{2} \mathrm{O}, 1: 1$ diluted with sea water), decapitated and heads transferred to a $1.5 \mathrm{ml}$ tube containing $150 \mu \mathrm{l}$ RIPA lysis buffer (R0278 Sigma-Aldrich), 10\% Triton X100 and protease inhibitor (cOmplete Tablets, EDTA-free, EASYpack, Roche). The tissue was homogenized by grinding using a tight fitting pestle. All steps on ice. Cell debris was pelleted by centrifugation. Protein concentration of lysates was determined using Bradford reagent (BIORAD), subjected to SDS-gel electrophoresis (10\% Acrylamide) and transferred (Transferbuffer: $39 \mathrm{mM}$ Gylcine, $48 \mathrm{mM}$ Tris, $0.04 \%$ SDS, $20 \%$ MetOH) to a nitrocellulose membrane (Amersham $^{\mathrm{TM}}$ Protran $^{\mathrm{TM}}$ 0,45 $\mu \mathrm{m}$ NC, GE Healthcare Lifescience). Quality of transfer was checked by Ponceau-S (Sigma Aldrich) staining. After $1 \mathrm{~h}$ of blocking with $5 \%$ slim milk powder (Fixmilch Instant, Maresi) in 1XPTW (1XPBS/0.1\% TWEEN 20) at room temperature, the membrane was incubated with the appropriate primary antibody diluted in $2.5 \%$ milk/PTW at $4^{\circ} \mathrm{C}$ overnight. [anti-L-Cry 5E3-3E6-E8 (1:100) and anti-L-Cry 4D4-3E12-E7 (1:100); anti-beta-Actin (Sigma, A-2066, 1: 20.000)]. After 3 rinses with 1xPTW the membrane was incubated with the species specific secondary antibody [anti-Mouse IgG-Peroxidase antibody, (Sigma, A4416, 1:7500); Anti-rabbit IgG-HRP-linked antibody (Cell Signaling Technology, \#7074, 1:7.500] diluted in 1xPTW/1\% slim milk powder for 1 hour, RT. After washing, SuperSignal ${ }^{\mathrm{TM}}$ West Femto Maximum Sensitivity Substrate kit (Thermo Fisher Scientific) was used for HRP-signal detection and finally signals were visualized by ChemiDoc Imaging System (BIORAD). Specific protein bands were quantified in "Image J" and L-Cry was normalized to beta-Actin.

\section{Collection and analysis of spawning data}

Worm boxes were checked daily for mature worms. Worms which had metamorphosed into their sexually mature male or female form and had left their tube to perform their nuptial dance were scored as mature animals.

The recordings of mature animals (collected from June 1929 to June 1930 in Naples $(28,29)$ ) were digitalized and all months were aligned to moonphase and combined. For comparisons of these data with our spawning data from the lab, we aligned the first day after full moon in nature with the last day of full moon stimulus in the lab, since Platynereis dumerilii synchronizes its circalunar clock to the 
712

713

714

715

716

717

718

719

720

721

722

723

724

725

726

727

728

729

730

731

732

733

734

735

736

737

738

739

740

moon off stimulus(5). For the circular analysis of spawning data, the lunar day of spawning was multiplied by 12 for each worm, so that the 30 lunar days regularly distributed on the $360^{\circ}$ circle. Circular analysis of these data was performed using Oriana (Version 4.02, Kovach Computing Services).

\section{Phase-Shift Experiments}

For Phase-Shift experiments, boxes with adult worms (at least 3 months old) were transferred from standard light conditions (see "worm culture") to the naturalistic light systems (sun- and moonlight) mounted in light-tight black shelves. Number of mature worms was recorded daily and number of mature worms per day was used to calculate percentage of mature worms (one month: 100\%). Data were smoothened using a rolling mean with a window size of 3 days. Weekly samplings of premature heads for gene expression analysis were performed at zt6 ( 2 heads per biological replicate) as described in "RNA extraction and RT-qPCR".

\section{RNA extraction and RT-qPCR}

For RNA expression analysis, worm heads were sampled at zt6 (6 hours after light on) by first anaesthetizing them for $5-10 \mathrm{~min}$ with a $1: 1$ mixture of seawater and $7.5 \%(\mathrm{w} / \mathrm{v}) \mathrm{MgCl}_{2}$ solution. The head was then cut behind the posterior eyes with a scalpel. 2-5 heads (depending on size) per biological replicate were collected in $2 \mathrm{ml}$ tubes containing metal beads, immediately frozen in liquid nitrogen and stored at $-80^{\circ} \mathrm{C}$ until RNA extraction.

For RNA extraction, 350 $\mu$ l of RNAzol RT (Sigma-Aldrich) were added to the samples and lysis was performed with Tissuelyser II (Qiagen) at $30 \mathrm{~Hz}$ for $2 \mathrm{~min}$. Afterwards, RNA was extracted using Directzol RNA Miniprep kit (Zymo Research) following the manufacturer's instructions with additional oncolumn DNasel digest. RNA was eluted in $34 \mu \mathrm{l}$ of nuclease-free water.

Total RNA (200ng per sample) was reverse transcribed using LunaScript RT SuperMix Kit (New England Biolabs). Final cDNA reactions were diluted to a final volume of 60-120 $\mu \mathrm{l}$. qPCR reactions were performed in $20 \mu \mathrm{l}$ total volume with Luna Universal qPCR Master Mix (New England Biolabs). Target genes and reference controls were analysed in duplicate reactions for all samples. Plate control cDNA and -RT controls were included on each plate. $c d c 5$ was used as reference gene (14, 25). Expression levels were calculated using the $\Delta c t$ method. Relative expression values were calculated with the formula: relative expression $=2^{-\Delta c t}$.

\begin{tabular}{|l|l|}
\hline Primer & Sequence 5' -3' $^{\prime}$ \\
\hline erp4.9_fwd & CAGAAGACCGTCACCAATGG \\
\hline erp4.9_rev & CTCGGTAATCTTCAGGCTAGGA \\
\hline g3bp2_fwd & TGAAATCCAGGAGGAAGTTGA \\
\hline
\end{tabular}




\begin{tabular}{|l|l|}
\hline g3bp2_rev & GGAGGATCGGGAGACACTATC \\
\hline cdc5_fwd & CCTATTGACATGGACGAAGATG \\
\hline cdc5_rev & TTCCCTGTGTGTTCGCAAG \\
\hline
\end{tabular}

\section{2}

Primers used for $q P C R$

743 RNA from immature worm heads was extracted and reverse transcribed (see "RNA extraction and

744 RT-qPCR" for details). PCR for amplification was performed using Phusion Polymerase (NEB). After gel 745 electrophoresis, DNA was extracted using the QIAquick Gel Extraction Kit (Qiagen) according to the 746 manufacturer's instructions and cloned into pJET1.2 using the CloneJET PCR Cloning Kit 747 (ThermoScientific). Clones were sequence verified and sequences submitted to GenBank (MT795155,

748 MT795156). Following sequences were used for phylogenetic trees in S7:

\begin{tabular}{|l|l|}
\hline Sequence & GenBankID \\
\hline Mmu-G3BP1 & P97855 \\
\hline Mmu-G3BP2 & P97379 \\
\hline Hsa-G3BP1 & Q13283 \\
\hline Hsa-G3BP2 & Q9UN86 \\
\hline Dre-G3BP2 & A1L1U3 \\
\hline Dre-G3BP1 & Q7ZVG3 \\
\hline Dme-RIN & Q9NH72 \\
\hline Aca-G3BP & XP_005110089 \\
\hline Aca-G3BP & XP_005110087 \\
\hline Ame-G3BP & XP_623996 \\
\hline Cgi-G3BP & XP_011454471.1 \\
\hline Pma-G3BP & XP_033741132 \\
\hline Nve-G3BP & XP_001641827 \\
\hline Aqu-G3BP & XP_019860730 \\
\hline Pdu-G3BP1/2 & MT795155 \\
\hline
\end{tabular}

GenBank IDs used for phylogenetic tree in S7B

\begin{tabular}{|l|l|}
\hline Sequence & GenBankID \\
\hline Mmu-YTHDF1 & P59326 \\
\hline Mmu-YTHDF2 & Q91YT7 \\
\hline Mmu-YTHDF3 & Q8BYK6 \\
\hline Hsa-YTHDF1 & Q9BYJ9 \\
\hline Hsa-YTHDF2 & Q9Y5A9 \\
\hline Hsa-YTHDF3 & Q7Z739 \\
\hline Dre-YTHDF1 & Q802Z0 \\
\hline Dre-YTHDF2 & E7F1H9 \\
\hline Dre-YTHDF3 & E7FAP0 \\
\hline Dme-YTHDF & Q9VBZ5 \\
\hline Cgi-YTHDF & XP_034317507 \\
\hline Ame-YTHDF & XP_624228 \\
\hline Aca-YTHDF & XP_005091424 \\
\hline Aqu-YTHDF & XP_003386824 \\
\hline Ami-YTHDF & XM_029351330 \\
\hline
\end{tabular}




\begin{tabular}{|l|l|}
\hline Primer & Sequence 5'-3' \\
\hline Pdu_g3bp2_F & TATCAAAGTCATGGTCATGGAAACTCCTAGC \\
\hline Pdu_g3bp2_R & TTTGATGTGATCCTCAAGGCCTAGCGC \\
\hline Pdu_ythdf2_F & AGAAACTGTGATGTCTGCAAGTGTAGACC \\
\hline Pdu_ythdf2_R & GGGCGCCACCTAGTTGCTCTGG \\
\hline
\end{tabular}

753

jetlag EST- GenBank MW161055

\section{Whole mount in situ Hybridisation combined with immunohistochemistry for L-Cry}

Probes were generated de novo using previously cloned plasmids as template (see "Cloning of g3bp1/2 and $y$ thdf1/2/3"). Genes of interest were amplified via PCR using Phusion Polymerase (NEB) and primers for pJET 1.2 with an overhang of Sp6 promoter. PCR product was purified following the protocol for "QIAquick PCR Purification Kit" (Qiagen). Agarose gel electrophoresis showed right amplicon sizes and single bands. For the in vitro transcription, $1 \mu \mathrm{g}$ of linearized template was used. Riboprobes were labelled with anti-Digoxigenin UTPs (Roche Diagnostics) and transcribed with Sp6 polymerase at $37^{\circ} \mathrm{C}$ for $4 \mathrm{~h}$. Probes were purified according to the "RNeasy Kit" (QIAGEN) and eluted in 40ul RNase-free water. 600-1000ng of the riboprobes were used.

Whole-mount in situ Hybridisation was carried out on premature worms of the RE strain, following published procedures $(47,50)$ with adjustments made to combine it with L-Cry immunohistochemistry. Worm heads were fixed in $4 \%$ PFA for $2 \mathrm{~h}$ at RT while shaking. Proteinase $\mathrm{K}$ digest: $5 \mathrm{~min}$, during which samples were very slightly rocked. After blocking in $5 \%$ sheep serum/1X PTW, worm heads were incubated with the monoclonal L-Cry antibodies (5E3-3E6-E8 diluted 1:100 and 4D4-3E12-E7 diluted 1:50), anti-Digoxigenin-AP coupled antibody (Roche Diagnostics) and sheep serum diluted to $2.5 \%$ with $1 \times$ PTW for $36-40 \mathrm{~h}$ at $4^{\circ} \mathrm{C}$, shaking. After detection using NBT/BCIP, samples were incubated in the secondary antibody Alexa Fluor-488 goat anti-mouse IgG (Thermo Fisher Scientific), 1:400 (36-40h at $4^{\circ} \mathrm{C}$, shaking), washed in 1xPTW and mounted in DABCO/Glycerol. Imaging was done using Axioplan Z2 Microscope (Carl Zeiss) with AxioCam MRc5 colour CCD camera (Carl Zeiss) and captured using ZenPro Software (Carl Zeiss). The images were edited with either ImageJ or Photoshop CC. 


\section{Treatment with C108 Compound}

Premature I-cry +/+ worms were kept in isolated shelves with 16:8 circadian light-dark regime and dim-moonlight conditions corresponding to the circalunar light schedule (8 nights of FM). Toxicity tests were conducted using increasing concentrations of the G3BP2 inhibitor, Compound C108 (Sigma Aldrich)(31). Comparison between the different concentrations showed reliable effects using 10uM of the drug while health and behaviour of worms overall seemed to be unaffected. Five days prior to taking the samples, cohorts of worms were treated with $10 \mu \mathrm{M}$ Compound C108 (SigmaAldrich). Control worms were cultured in parallel and treated with corresponding amounts of DMSO. Sampling (at zt6, two heads per biological replicate) and RT-qPCR was performed as described in "RNA extraction and RT-qPCR".

\section{Statistical Analysis}

All data analysis was conducted using R 3.6.1(43), GraphPad Prism 8.4.2, Oriana 4.02 and Microsoft Excel 2010.

\section{Acknowledgements:}

We thank the members of the Tessmar-Raible and Wolf groups for discussions. Andrej Belokurov and Margaryta Borysova for excellent worm care at the MFPL aquatic facility. We are grateful for support by the IMB Protein Production and Proteomics Core Facilities (mass spec instrument funded by DFG INST 247/766-1 FUGG). In particular, we wish to thank Dr. Mario Dejung of the Proteomics Core Facility for carrying out preliminary mass spec data analysis. We would like to thank Dr Svenja Morsbach and Beate Müller from the Max Planck Institute for Polymer Research for helping with HPLC experiment and Prof Dr Elmar Jaenicke from University of Mainz for help with SEC-MALS.

\section{Funding}

K.T-R. received funding for this research from the European Research Council under the European Community's Seventh Framework Programme (FP7/2007-2013) ERC Grant Agreement 337011 and the Horizon 2020 Programme ERC Grant Agreement 819952, the research platform 'Rhythms of Life' of the University of Vienna, the Austrian Science Fund (FWF, http://www.fwf.ac.at/en/): SFB F78 and the HFSP () research grant (\#RGY0082/2010). S.K. is a recipient of a DFG fellowship through the Excellence Initiative by the Graduate School Materials Science in Mainz (GSC 266). None of the funding bodies was involved in the design of the study, the collection, analysis, and interpretation of data or in writing the manuscript.

\section{References:}

1. H. M. Fox, Lunar periodicity in Reproduction. Proceedings of the Royal Society of London Series B 95, 523-550 (1924). 
809 2. O. Levy et al., Light-responsive cryptochromes from a simple multicellular animal, the coral Acropora millepora. Science 318, 467-470 (2007).

811 3. H. Numata, B. Helm, Annual, lunar, and tidal clocks : patterns and mechanisms of nature's enigmatic rhythms. (Springer, Tokyo ; New York, 2014), pp. xii, 360 pages.

814

4. P. Korringa, Relations between the moon and periodicity in the breeding of marine animals. Ecological Monographs 17, 347-381 (1947).

5. C. Hauenschild, Lunar periodicity. Cold Spring Harb. Symp. Quant. Biol. 25, 491-497 (1960).

6. K. Tessmar-Raible, F. Raible, E. Arboleda, Another place, another timer: Marine species and the rhythms of life. Bioessays 33, 165-172 (2011).

7. V. S. Bézy et al., Mass-nesting events in olive ridley sea turtles: environmental predictors of timing and size. Anim. Behav. 163, 85-94 (2020).

8. G. Norevik, S. Akesson, A. Andersson, J. Backman, A. Hedenstrom, The lunar cycle drives migration of a nocturnal bird. PLoS Biol. 17, e3000456 (2019).

9. L. Casiraghi et al., Moonstruck sleep: Synchronization of human sleep with the moon cycle under field conditions. Sci Adv 7, (2021).

10. C. Helfrich-Forster et al., Women temporarily synchronize their menstrual cycles with the luminance and gravimetric cycles of the Moon. Sci Adv 7, (2021).

11. T. Shlesinger, Y. Loya, Breakdown in spawning synchrony: A silent threat to coral persistence. Science 365, 1002-1007 (2019).

12. D. Neumann, Temperature compensation of circasemilunar timing in the intertidal insect Clunio. Journal of Comparative Physiology A - Sensory Neural and Behavioral Physiology 163, 671-676 (1988).

13. H.-D. Franke, The Role of Light and Endogenous Factors in the Timing of the Reproductive Cycle of Typosyllis prolifera and Some Other Polychaetes. Am. Zool. 26, 433-445 (1986).

14. J. Zantke et al., Circadian and Circalunar Clock Interactions in a Marine Annelid. Cell Reports 5, 99-113 (2013).

15. H. D. Franke, On a clocklike mechanism timing lunar-rhythmic reproduction inTyposyllis prolifera (Polychaeta). Journal of Comparative Physiology A: Neuroethology, Sensory, Neural, and Behavioral Physiology 156, 553-561 (1985).

16. F. Raible, H. Takekata, K. Tessmar-Raible, An Overview of Monthly Rhythms and Clocks. Frontiers in Neurology 8, (2017).

17. C. Hauenschild, Über das lunarperiodische Schwärmen von Platynereis dumerilii in Laboratoriumszuchten. Naturwissenschaften 41, 556-557 (1954).

18. K. Fukunaga, F. Yamashina, Y. Takeuchi, C. Yamauchi, A. Takemura, Moonlight is a key entrainer of lunar clock in the brain of the tropical grouper with full moon preference. $B M C$ Zoology 5, 11 (2020).

19. M. Fukushiro et al., Lunar phase-dependent expression of cryptochrome and a photoperiodic mechanism for lunar phase-recognition in a reef fish, goldlined spinefoot. PLOS ONE 6, e28643 (2011).

20. A. K. Brady, B. L. Willis, L. D. Harder, P. D. Vize, Lunar Phase Modulates Circadian Gene Expression Cycles in the Broadcast Spawning Coral Acropora millepora. Biol. Bull. 230, 130142 (2016).

21. A. Berndt et al., A novel photoreaction mechanism for the circadian blue light photoreceptor Drosophila cryptochrome. J. Biol. Chem. 282, 13011-13021 (2007).

22. V. B. Veedin Rajan et al., Seasonally relevant UVA light alters neurohormone amounts and behavior via a ciliary opsin in a marine mass spawning annelid. Nature Ecology \& Evolution, (2021).

23. S. Bannister et al., TALE Nucleases mediate efficient, heritable genome modifications in the marine annelid Platynereis dumerilii. Genetics 197, 19-31 (2014).

24. B. Xu, X. Feng, R. D. Burdine, Categorical data analysis in experimental biology. Dev. Biol. 348, 3-11 (2010).

25. S. Schenk et al., Combined transcriptome and proteome profiling reveals specific molecular brain signatures for sex, maturation and circalunar clock phase. Elife 8, (2019). 
26. T. Ayers, H. Tsukamoto, M. Gühmann, K. Tessmar-Raible, A Go-type Opsin mediates the Shadow Reflex in the annelid Platynereis dumerilii. BMC Biol. 16, 41 (2018).

27. M. Guehmann et al., Spectral Tuning of Phototaxis by a Go-Opsin in the Rhabdomeric Eyes of Platynereis. Curr. Biol. 25, 2265-2271 (2015).

28. S. Ranzi, Ricerche sulla biologia sessuale degli Anellidi. Pubbl. Staz .Zool. Napoli 11, 271-292 (1931).

29. S. Ranzi, Maturita sessuale degli Anellidi e fasi lunari. . Boll. Soc. Ital. Biol. Sperim. 6, 18 (1931).

30. J. Zantke, S. Bannister, V. B. Veedin Rajan, F. Raible, K. Tessmar-Raible, Genetic and Genomic Tools for the marine annelid Platynereis dumerilii. Genetics 197, 9-31 (2014).

31. N. Gupta et al., Stress granule-associated protein G3BP2 regulates breast tumor initiation. Proc. Natl. Acad. Sci. U. S. A. 114, 1033-1038 (2017).

32. N. Peschel, K. F. Chen, G. Szabo, R. Stanewsky, Light-Dependent Interactions between the Drosophila Circadian Clock Factors Cryptochrome, Jetlag, and Timeless. Curr. Biol. 19, 241247 (2009).

33. U. Alam, D. Kennedy, Rasputin a decade on and more promiscuous than ever? A review of G3BPs. Biochim Biophys Acta Mol Cell Res 1866, 360-370 (2019).

34. J. D. Laver et al., The RNA-Binding Protein Rasputin/G3BP Enhances the Stability and Translation of Its Target mRNAs. Cell Rep 30, 3353-3367 e3357 (2020).

35. S. Zaccara, S. R. Jaffrey, A Unified Model for the Function of YTHDF Proteins in Regulating m(6)A-Modified mRNA. Cell 181, 1582-1595 e1518 (2020).

36. B. Collins, E. O. Mazzoni, R. Stanewsky, J. Blau, Drosophila CRYPTOCHROME is a circadian transcriptional repressor. Curr. Biol. 16, 441-449 (2006).

37. A. Abakir et al., N(6)-methyladenosine regulates the stability of RNA:DNA hybrids in human cells. Nat. Genet. 52, 48-55 (2020).

38. U. Kubitscheck, J.-P. Siebrasse, Kinetics of transport through the nuclear pore complex. Semin. Cell Dev. Biol. 68, 18-26 (2017).

39. A. Czarna et al., Structures of Drosophila Cryptochrome and Mouse Cryptochrome1 Provide Insight into Circadian Function. Cell 153, 1394-1405 (2013).

40. N. S. Häfker, K. Tessmar-Raible, Rhythms of behavior: are the times changin'? Curr. Opin. Neurobiol. 60, 55-66 (2020).

41. G. Andreatta, K. Tessmar-Raible, The Still Dark Side of the Moon: Molecular Mechanisms of Lunar-Controlled Rhythms and Clocks. J. Mol. Biol., (2020).

42. P. Oliveri et al., The Cryptochrome/Photolyase Family in aquatic organisms. Marine Genomics 14, 23-37 (2014).

43. R. C. Team, in R Foundation for Statistical Computing. (https://www.R-project.org/Vienna, Austria, 2016).

44. J. Cox, M. Mann, MaxQuant enables high peptide identification rates, individualized p.p.b.range mass accuracies and proteome-wide protein quantification. Nat. Biotechnol. 26, 13671372 (2008).

45. J. L. Hsu, S. Y. Huang, N. H. Chow, S. H. Chen, Stable-isotope dimethyl labeling for quantitative proteomics. Anal. Chem. 75, 6843-6852 (2003).

46. C. Hauenschild, A. Fischer, "Platynereis dumerilii. Mikroskopische Anatomie, Fortpflanzung, Entwicklung. [Platynereis dumerilii. Microscopical anatomy, reproduction and development] " Großes Zoologisches Praktikum Heft. (Stuttgart, 1969).

47. K. Tessmar-Raible, P. R. Steinmetz, H. Snyman, M. Hassel, D. Arendt, Fluorescent two-color whole mount in situ hybridization in Platynereis dumerilii (Polychaeta, Annelida), an emerging marine molecular model for evolution and development. Biotechniques 39, 460, 462, 464 (2005).

48. J. Schindelin et al., Fiji: an open-source platform for biological-image analysis. Nat. Methods 9, 676-682 (2012).

49. C. Stringer, T. Wang, M. Michaelos, M. Pachitariu, Cellpose: a generalist algorithm for cellular segmentation. Nat. Methods 18, 100-106 (2021). 
bioRxiv preprint doi: https://doi.org/10.1101/2021.04.16.439809; this version posted April 18, 2021. The copyright holder for this preprint (which was not certified by peer review) is the author/funder, who has granted bioRxiv a license to display the preprint in perpetuity. It is made available under aCC-BY-NC-ND 4.0 International license.

50. B. Backfisch et al., Stable transgenesis in the marine annelid Platynereis dumerilii sheds new light on photoreceptor evolution. Proc Natl Acad Sci USA 110, 193-198 (2013).

917 